\title{
Robust, Practical Adaptive Control for Launch Vehicles
}

\author{
Jeb S. Orr* \\ Science Applications International Corporation, Huntsville, AL, 35806 \\ Tannen S. VanZwieten ${ }^{\dagger}$ \\ NASA Marshall Space Flight Center, AL, 35812
}

\begin{abstract}
A modern mechanization of a classical adaptive control concept is presented with an application to launch vehicle attitude control systems. Due to a rigorous flight certification environment, many adaptive control concepts are infeasible when applied to high-risk aerospace systems; methods of stability analysis are either intractable for high complexity models or cannot be reconciled in light of classical requirements. Furthermore, many adaptive techniques appearing in the literature are not suitable for application to conditionally stable systems with complex flexible-body dynamics, as is often the case with launch vehicles.

The present technique is a multiplicative forward loop gain adaptive law similar to that used for the NASA $\mathrm{X}-15$ flight research vehicle. In digital implementation with several novel features, it is well-suited to application on aerodynamically unstable launch vehicles with thrust vector control via augmentation of the baseline attitude/attitude-rate feedback control scheme. The approach is compatible with standard design features of autopilots for launch vehicles, including phase stabilization of lateral bending and slosh via linear filters. In addition, the method of assessing flight control stability via classical gain and phase margins is not affected under reasonable assumptions. The algorithm's ability to recover from certain unstable operating regimes can in fact be understood in terms of frequency-domain criteria. Finally, simulation results are presented that confirm the ability of the algorithm to improve performance and robustness in realistic failure scenarios.
\end{abstract}

\section{Introduction}

While industry practices for launch vehicle control system design have seen very little variation since the advent of the ballistic missile era, the academic community has been emphasizing the development of a wide array of modern control techniques for decades. Among the most promising of these is adaptive control. This type of advanced control has the ability to modify its characteristics on-line in response to unknown or unmodeled dynamics, nonlinearities, and environmental disturbances.

Adaptive control techniques (implemented predominantly using analog hardware) showed great promise in aerospace vehicle applications in the late 1950s and early 1960s, and saw numerous flight tests on experimental vehicles ${ }^{1,2}$. Adaptive control was primarily employed to offset low simulation model fidelity and the limited capabilities of ana$\log$ control systems. Soon thereafter, the widespread proliferation of the digital computer yielded a high-fidelity simulation-based design process. The performance and simplicity of scheduled digital gains and filters then cast a shadow on the early momentum of aerospace vehicle adaptive control systems, and largely relegated the theory and practice to academic interest.

While the underlying theory has been thoroughly developed and has yielded a rich body of peer-reviewed literature, modern adaptive techniques have resisted widespread adoption by the aerospace community. Despite the potential benefits, many academic approaches to adaptive control design present an over-generalized theory and lack cognizance of the essential features of real aerospace systems that challenge the practicing control design engineer. Reliance on advanced nonlinear stability analysis techniques that increase control gains in order to achieve asymptotic stability (or decrease error bounds) are not tractable for high-complexity aerospace systems. In addition, metrics of performance and stability for adaptive control systems are difficult to reconcile in the context of classical control system performance and robustness requirements such as gain and phase margin. Furthermore, many approaches are very computationally expensive and require complex algorithms that may challenge software implementation and introduce risk. Most importantly, many adaptive control systems are formulations that, although mathematically rigorous, appear as a "black box" to the risk-adverse systems engineer.

In order to fully realize the benefits of adaptive control for a particular application, the adaptive control system must be architected with an awareness of the total system to which it is to be applied. This includes unique features of the

${ }^{*}$ Flight Controls Engineer, Space Engineering Technology Division

${ }^{\dagger}$ Aerospace Engineer, Control Systems Design and Analysis Branch 
aerospace vehicle, such as control-structure interaction, propellant slosh, sensor performance, and actuator dynamics. In addition, the analysis, verification, and flight certification framework for the control system must be addressed. Launch vehicle applications (especially those with crew) are particularly challenging in this regard given their unique dynamics, the rigorous certification environment, and the enormous cost associated with an in-flight anomaly leading to the loss of a vehicle.

This paper focuses on adaptive control developments that are specifically tailored for application to launch vehicles. The control architecture is described, including measures that were taken to mitigate risk and maintain consistency with classical control system design and requirements verification. Simulation results for several credible launch vehicle failure scenarios are presented and discussed. For each of the documented test cases, high-fidelity launch vehicle analysis tools were employed in order to quantify and document specific benefits and risks of the proposed adaptive control scheme.

\section{Background}

For high risk aerospace applications, government and industry are very reliant on classical control theory. The most common type of feedback control system for launch vehicles is the proportional-integral-derivative (PID) controller with appropriate structural bending filters to effect active (phase) or passive (gain) stabilization of the elastic modes sensed by rate gyros located on the vehicle structure. Launch vehicles are often aerodynamically unstable, have relatively slow servoactuators, and may exhibit nonminimum phase characteristics due to non-collocation of the actuator and flight control sensors. Fortunately, launch vehicle dynamics are readily modeled using linear techniques whose limitations are well understood ${ }^{1,3-5}$. It follows that control system design, analysis, and verification can be approached in the frequency domain, yielding the gain and phase margin requirements typically used for flight certification.

In the case of launch vehicle flight controls, the continued use of classical methods is encouraged by a proven record of success. In fact, GN\&C issues are rarely the cause for launch vehicle failures. On the other hand, a large percentage of failures could have been prevented through the use of advanced GN\&C technologies. A review of historical launch vehicle data from 1990 to 2002 revealed that $41 \%$ of failures might have been mitigated by advanced GN\&C technologies ${ }^{6}$. Therefore, while classical control methods are typically able to meet requirements for flight, the development of advanced control designs have the potential to improve performance capabilities, add robustness, decrease the costs associated with high fidelity models and tests, and allow recovery from severely off-nominal, unanticipated scenarios. Even in cases where vehicle damage or degraded performance prevents mission success, adaptive control techniques may be able to delay an abort or range safety destruct event long enough to improve chances of crew survival and reduce the risk to property and the public.

\section{A. Ground Rules and Assumptions}

The aforementioned advantages of an adaptive control system present an obvious entry point for control architecture development leading to additional robustness for launch vehicles. The present approach seeks to integrate technological advancements with heritage control techniques through an Adaptive Augmenting Control (AAC) algorithm. This approach pairs an adaptive controller with a classically designed linear control system in such a way that it provides an augmenting action. The classical system retains the core feedback control functionality for stabilization and guidance command following. In such an architecture, the adaptive controller should provide little or no augmentation to the classical control system in the case that a given flight is within typical parameter and environment uncertainty bounds; that is, a well-designed linear controller should not require augmentation to provide acceptable performance in this case. However, in the event that the baseline controller is unable to provide sufficient control performance (due to parameter variation, external disturbances, or model error), the adaptive controller should have the ability to modify the output of the baseline feedback control law so as to recover the performance loss to the maximum extent possible.

The adaptive controller must consistently and predictably improve performance and robustness, and its behavior must be conceptually extensible to failure scenarios that cannot be tested, simulated, or even imagined. The adaptive element must operate within reasonable boundaries that allow for additional performance but prevent the adaptive controller from demanding more from the system than the physics allow. A conceptual depiction of the regions of operation of such an adaptive control system appears in Figure 1. 


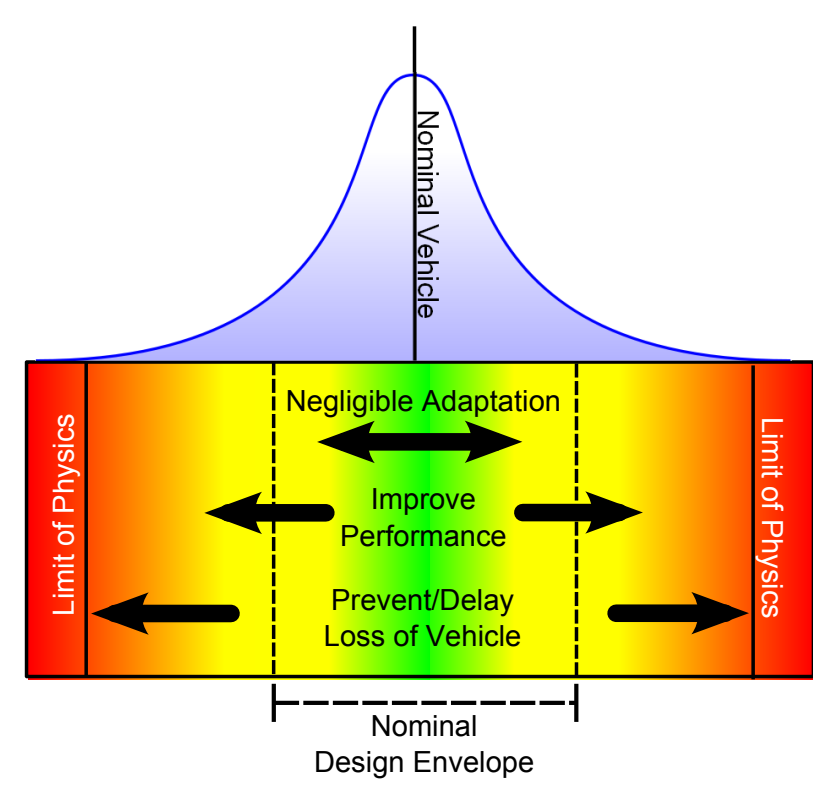

Figure 1. Conceptual regions of operation for adaptive augmentation

Finally, the adaptive controller must integrate as much as practicable with existing techniques for analyzing control stability and performance, including the determination of adaptation limits and their effect on classical stability margins. A clear path should exist to correlate, without the absolute requirement of an extensive and rigorous theory, the action of the adaptive controller with the linear time-invariant system characteristics currently used for flight certification.

\section{Control Architecture}

An architecture has been developed in-house at Marshall Space Flight Center (MSFC) by civil service and contractor personnel in the Flight Mechanics and Analysis Division (EV40). This design is extended from a series of heritage concepts, especially the MH-90 and MH-96 adaptive autopilots that were supported by Air Force research in the late 1950 s and early $1960 \mathrm{~s}^{7,8}$.

The fundamental principle of operation in the present system is an increase in system gain to minimize error with respect to a model reference, paired with a gain reduction capability based upon the frequency response characteristics of the closed-loop system. Other system features are included in the implementation to enforce strict adaptation limits that can be derived from the classical stability margins of the nominal system.

Single-axis attitude control of an aerospace vehicle yields an underlying second-order plant (the rigid-body attitude dynamics) with additional parasitic dynamics (such as actuator dynamics, slosh, and elastic response). In the ideal system, the precision control of the attitude states is separated from the parasitic dynamics in the frequency domain. As will be shown, the characteristics of the closed-loop spectrum can be determined in real time via filtering; the resultant signal can then be used in the adaptive control policy to effect a response to dynamics other than the controlled rigidbody motion.

The MH-96 forward gain-adaptive system, originally developed for the canceled X-20 program, is particularly interesting in that it was employed with great success on the X-15 rocket-propelled research aircraft. This system saw over 60 successful test flights and significantly improved performance and pilot opinion of aircraft handling qualities ${ }^{8}$. However, the MH-96 was designed to operate continuously at the stability limit and exhibited a failure mode that contributed to the loss of X-15-3 in $1967^{2}$. In particular, the analog implementation of the frequency-dependent component was subject to electrical saturation, ${ }^{\ddagger}$ rendering the system gain adaptation mechanism inoperable for large command signals ${ }^{7,8}$. In fact, the command signals contributing to the 1967 failure were the result of large pilot inputs.

By acting as an augmenting controller rather than the primary method of accommodating the changing flight envelope, the present design adheres to a significantly different paradigm. This shift in the concept of operation, the

${ }^{\ddagger}$ From ${ }^{8}$, p.21: ...the gain changer was misled by the direct-current or low-frequency signals... masking signals within the bandpass frequency range. The gain increased to values exceeding the critical gain, and the servoactuator loop became unstable. 
digital filter implementation's immunity to saturation nonlinearities, and additional protections afforded by the design of the adaptive law eliminates the failure modes that shadowed the otherwise highly successful X-15 adaptive control research effort.

\section{A. Classical Stability Margins and The Closed-Loop Spectrum}

Consider the classical PID-type feedback control system shown in Figure 2. With a fixed ratio of proportional and derivative gains, the system provides the highest possible performance at a high forward loop gain $k_{T}$. However, the unique stability characteristics (due to flexibility, servo lag, and aerodynamic instability) necessitates the choice of a small range of forward loop gains that provide performance and robustness for the baseline controller. Design requirements typically limit the permissible gain to not less than $6 \mathrm{~dB}$ from any critical value to protect for uncertainties. Uncertain parameters include the location of the center of mass with respect to each gimbal axis of rotation, the moment of inertia of the controlled axis, the thrust of each engine, the servo command gain, and the aerodynamic coefficient slope.

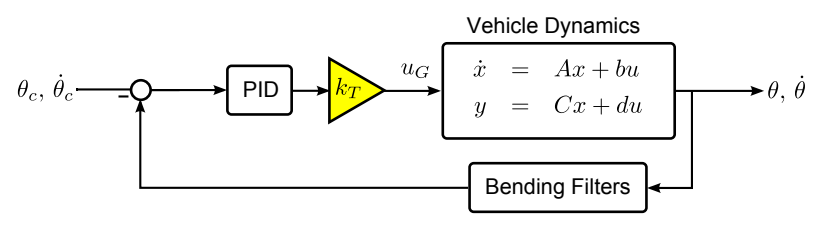

Figure 2. Baseline autopilot block diagram

The linear time-invariant (LTI) stability margins are computed at the gimbal servocommand input $\left(u_{G}\right)$ to autopilot output open loop, so chosen because the loop is single-input, single-output (SISO) and captures a plethora of robustness information in the frequency response. In addition to the aforementioned gain margins, designs typically require robustness of up to 30 degrees of phase lag at the first gain crossover frequency. Active or passively stabilized elastic modes usually require 30 to 45 degrees of phase margin, and 6 to $10 \mathrm{~dB}$ of gain margin, respectively, at frequencies within the control loop bandwidth.

An example of typical launch vehicle open-loop frequency-domain response appears in the log-magnitude (Nichols) chart in Figure 3. The depicted frequency response clearly shows an actively stabilized lateral bending mode. A critical stability limit at any high frequency (thus defining a gain margin) is the compound result of various factors, and differs based on the vehicle configuration. One often considers the high-frequency gain margins to be defined by the plant dynamics whose complex poles near that frequency first cross the imaginary axis in the closed-loop root locus under the effect of increasing forward loop gain. For the so-called rigid-body gain margin, these poles are most commonly those associated with the rigid body dynamics, and their behavior results from the filter and servo lag necessary to effect active stabilization of the first lateral bending mode. Alternatively, for relatively stiff vehicles with slow actuators, servo lag is the dominant factor and the servo poles may define the gain margin. Less commonly, lightly damped high-frequency passively stabilized elastic or propellant slosh modes may become unstable first under increasing gain. 


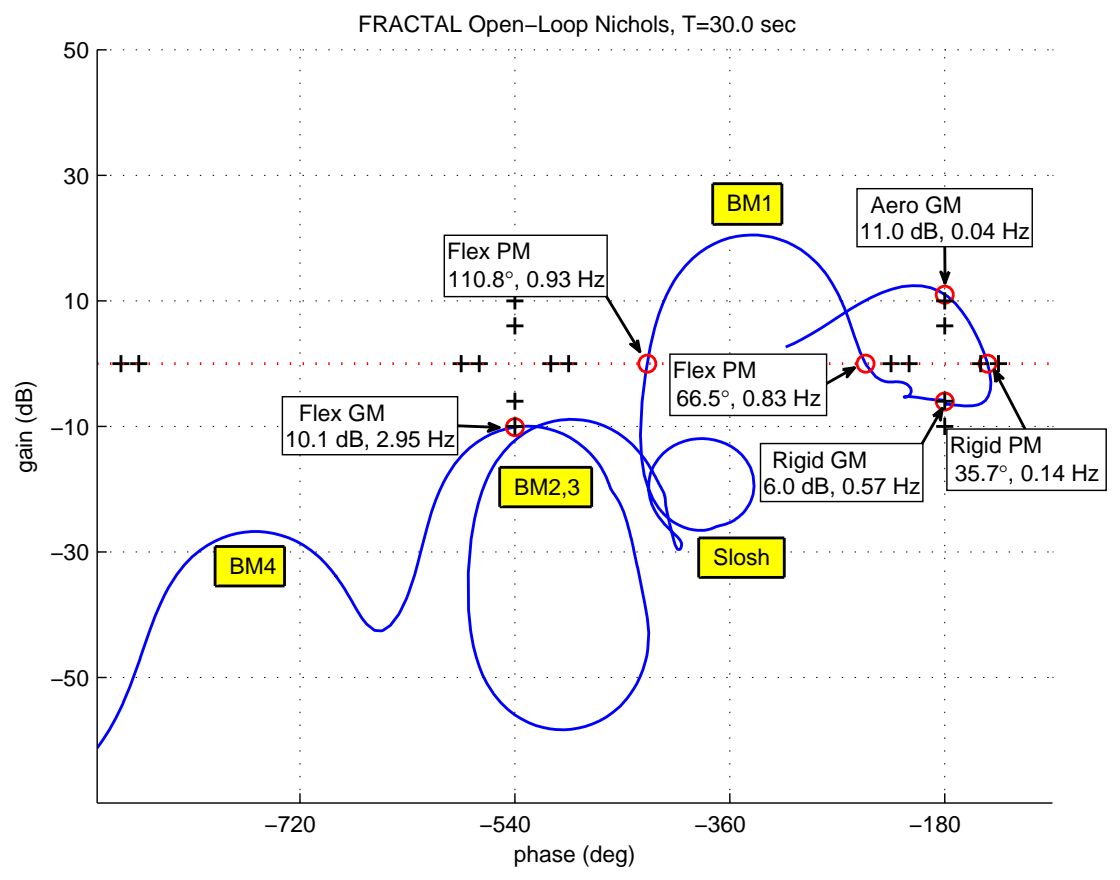

Figure 3. Typical launch vehicle Nichols open-loop response

The closed-loop spectrum, or the frequency response of the system under closed-loop control, is closely related to the open-loop margins. At the critical stability limit, the frequency of the open-loop spectrum closest to the $-1+j 0$ critical point is indicative of the frequency at which the poles of the associated dynamics will cross the $j \omega$ axis in the complex plane. At this limit with some critical forward loop gain $k_{T}$, the closed-loop system will exhibit resonance at this frequency and will display an unstable (divergent) mode at any gain $k_{T}+\epsilon, \epsilon>0$. An example of this phenomenon is shown in Figure 4, where the forward loop gain has been increased so as to drive the rigid-body poles at $f \approx 0.57$ $\mathrm{Hz}$ unstable. Knowledge of this behavior can yield a useful signal for on-line adjustment of the forward loop gain. 


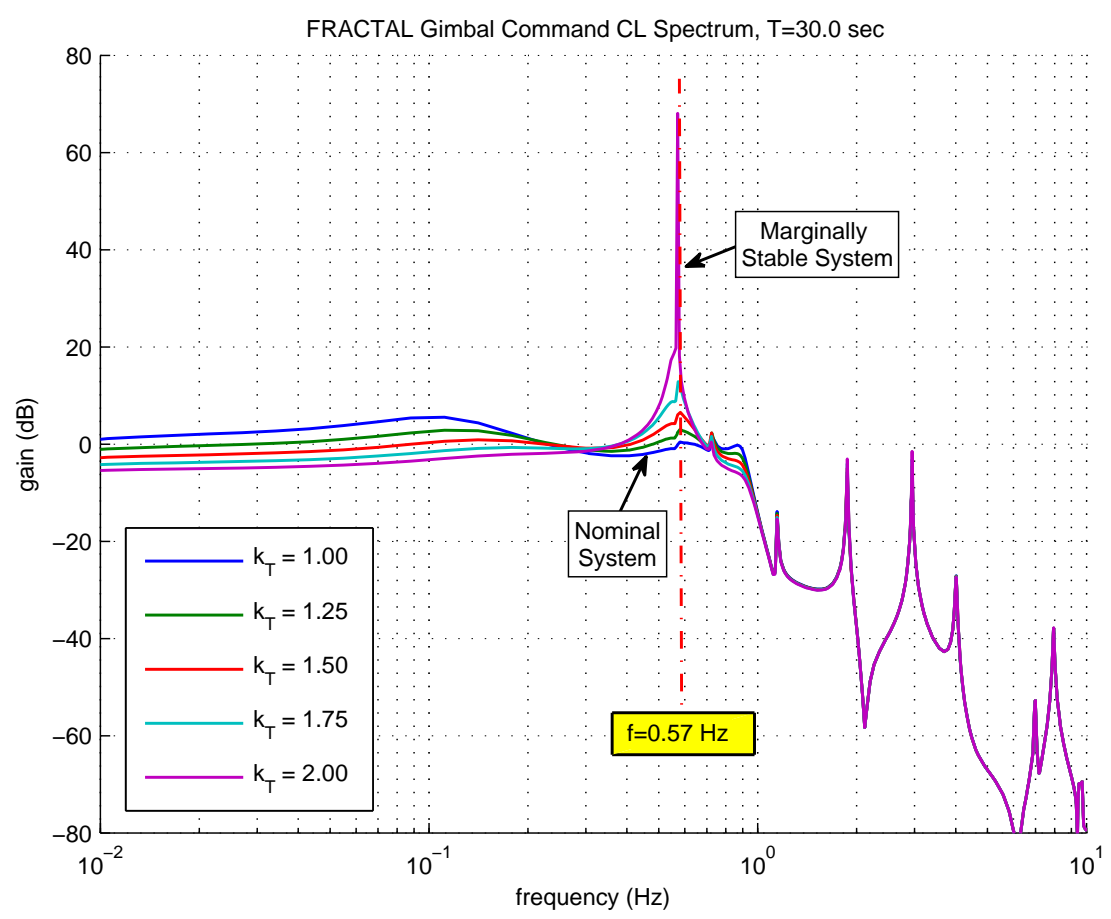

Figure 4. Forward loop gain instability

\section{B. Multiplicative Adaptive Control With Spectral Damping}

Historical forward loop gain adaptive systems ${ }^{1,7}$ relied upon the known frequency response characteristics of the servoactuator, which could easily be modeled and tested, to drive the system to a stable limit cycle of a prescribed amplitude at which the highest possible performance could be attained. This was accomplished by increasing the forward loop gain until the system exhibited limit cycle behavior. In the case where the limiting factor was the servo dynamics, the limit cycle frequency was associated with the second-order angular dynamics of the control surface or actuator. In the more general case, the limiting factor may be at any frequency above the rigid body control frequency.

\section{Adaptive Gain Law}

The present design uses a multiplicative first-order adaptation law and is implemented using the architecture shown in Figure 5. A multiplicative law is chosen rather than an additive law due to the predictable effect of adaptation on the gain margin. Under the assumption of decoupled yaw, pitch, and roll control, one adaptation law is used per axis. The output of the adaptive law is the adaptive gain, $k_{a}$, which is used to multiplicatively adjust the output of the baseline controller. The baseline controller is a PID-type controller with linear bending filters, assumed to be already be designed optimally for the nominal ensemble of vehicles. The baseline controller, therefore, always provides the basic feedback control action. The adaptive gain $k_{a}$ is used to compute a total multiplicative forward loop gain given by

$$
k_{T}=k_{0}+k_{a}
$$

that ranges from the minimum gain $k_{0}>0$ to some upper bound established by prescribing a limit on $k_{a}$. Both the upper and lower bounds can be determined from the classical gain margins for the nominal system model. For example, if the nominal system model under feedback control has an open-loop gain margin of $6 \mathrm{~dB}$ and $k_{0}=0.5$, the maximum $k_{a}$ is chosen to be 1.5 . This yields an upper limit for $k_{T}$ of 2 , or approximately $6 \mathrm{~dB}$. While the gain limits are chosen based on the nominal system model, the mechanism of adaptation relies more heavily on other features to increase robustness and limit adaptation. 


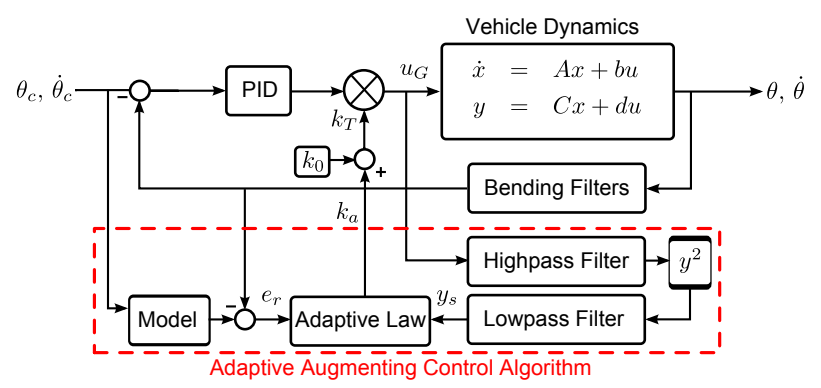

Figure 5. Baseline autopilot with adaptive augmentation

The adaptation law is governed by the solution of a first-order, nonlinear, ordinary differential equation (ODE), and includes a natural damping or first-order leakage term, limits on the upper and lower bounds of the adaptive variable, and a special filtered error signal called a spectral damper that responds to undesirable high-frequency dynamics.

The dynamics of the adaptive gain are given by

$$
\dot{k}_{a}=\left(\frac{k_{\max }-k_{a}}{k_{\max }}\right) a e_{r}^{2}-\alpha k_{a} y_{s}-\beta\left(k_{T}-1\right)
$$

where $a$ is the adaptive error gain, $\alpha$ is the spectral damper gain, and $\beta$ is the leakage gain.

\section{Computation of Reference Model Error}

The reference model error signal $e_{r}$ is the only mechanism that increases the adaptive gain. This error is computed as a linear combination of the magnitude and rate of the reference model tracking error, given by

$$
e_{r}=c \gamma_{r}+\dot{\gamma}_{r}
$$

with $c>0$ and $\gamma_{r}=\theta_{r}-\theta$. The reference model states $\theta_{r}$ and $\dot{\theta}_{r}$ are calculated on-line for use in deriving the reference model error. Both states are determined from a simple second-order reference model with a time-varying natural frequency, given by

$$
\left[\begin{array}{c}
\dot{\theta}_{r} \\
\ddot{\theta}_{r}
\end{array}\right]=\left[\begin{array}{cc}
0 & 1 \\
-\omega_{r}^{2} & -2 \zeta_{r} \omega_{r}
\end{array}\right]\left[\begin{array}{c}
\theta_{r} \\
\dot{\theta}_{r}
\end{array}\right]+\left[\begin{array}{cc}
0 & 0 \\
\omega_{r}^{2} & 2 \zeta_{r} \omega_{r}
\end{array}\right]\left[\begin{array}{c}
\theta_{c} \\
\dot{\theta}_{c}
\end{array}\right]
$$

where $\theta_{c}, \dot{\theta}_{c}$ are the guidance-commanded attitude and rate, respectively. The error mixing constant $c$ is chosen considering that in a high gain mode of operation, $e_{r} \approx 0$ and the error dynamics are approximately governed by

$$
\dot{\gamma}_{r}=-c \gamma_{r}
$$

which converges asymptotically to zero with time constant $1 / c$. The time constant is chosen to be of the same order as the rigid-body attitude dynamics. In implementation, the closed-form discretization of the state model (2) at the control update rate is accomplished using standard methods.

\section{Logistic Gain Limiting}

The adaptive gain $a$ is preceded by a normalized saturation term similar to the well-known logistic equation, a common model for certain classes of physical systems. The logistic term enforces a smooth, continuous upper limit to the adaptive gain $k_{a}$ by decreasing the adaptation rate to zero as $k_{a} \rightarrow k_{\max }$. The upper gain limit is determined from linear stability analysis of the nominal system, and can be gain-scheduled with the remainder of the control system parameters so as to provide a variable limit to the available authority of the adaptive controller at various points along the trajectory. Since the nominal system is used to choose the limit, $k_{\max }$ may be set by the designer at a value slightly above the critical stability limit for the nominal vehicle. This choice affords the adaptive controller maximum latitude in the event of an unanticipated decrease in effective forward loop gain or large external disturbances. 


\section{Spectral Damper}

The spectral damping gain $\alpha$ is used to introduce the spectral damper output signal $y_{s}$, which is formed from the controller gimbal command output $u_{G}$ as

$$
\begin{aligned}
y_{H P} & =H_{H P}(s) u_{G} \\
y_{s} & =H_{L P}(s) y_{H P}^{2}
\end{aligned}
$$

where $H_{H P}$ is a linear band-pass or high-pass filter and $H_{L P}$ is a linear low-pass filter. Note that the filtering process (3) rectifies the high-frequency components of $u_{G}$, the controller output. The action of the spectral damping signal $y_{s}$ mitigates instability by reducing excessive forward loop gain so as to prevent control-structure interaction observed in the control signal itself. If $k_{0}<1$, the adaptation can reduce the controller gain below the nominal.

In general, $H_{H P}$ is designed to have a DC gain as small as possible, a passband ${ }^{\S}$ gain of unity, and a steep transition band with the $-3 \mathrm{~dB}$ bandwidth occurring approximately one octave above the rigid-body control frequency. The lowpass filter $H_{L P}$ is chosen to be a maximally flat filter with a $-3 \mathrm{~dB}$ bandwidth approximately matching the rigidbody control frequency. Examples of the magnitude response for the band-pass and low-pass filters appear in Figure 6.
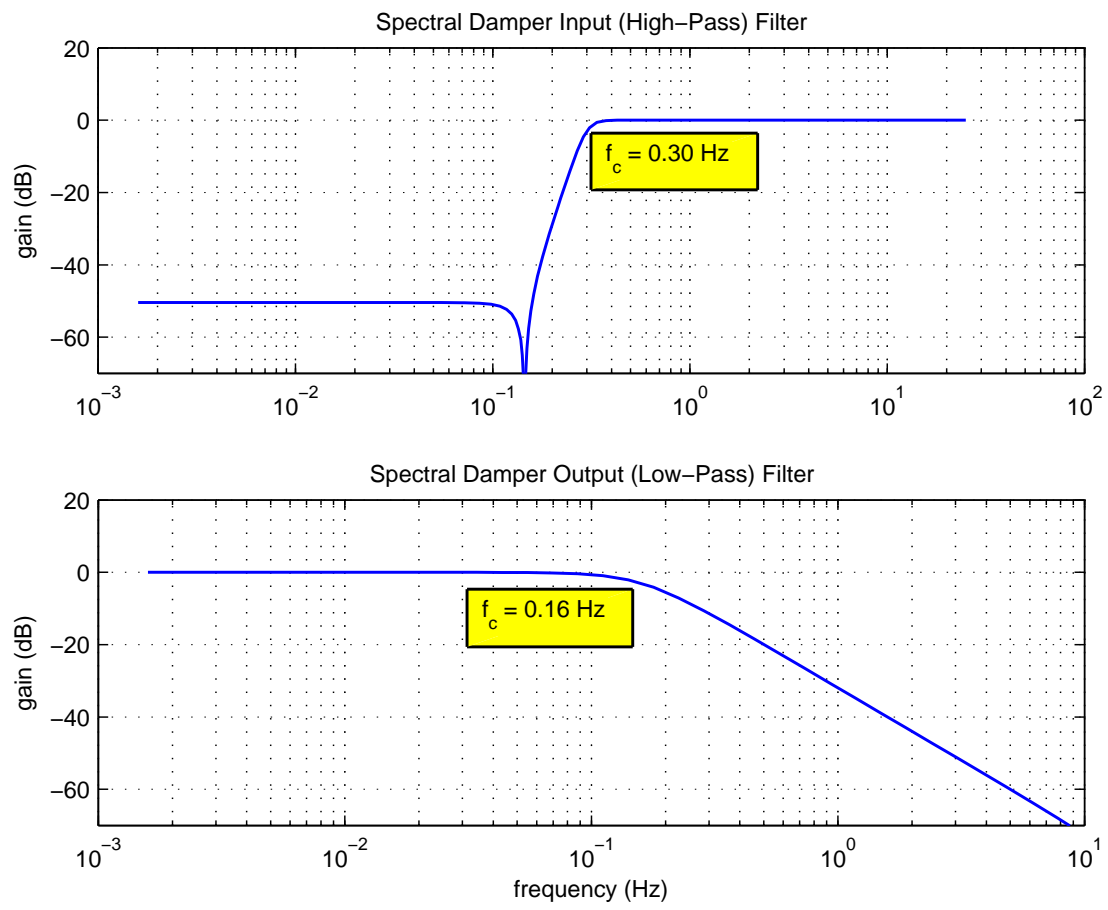

Figure 6. Spectral damper filter magnitude response

Additional design flexibility for the input filter is available with the consideration that the first lateral bending mode is actively stabilized in the baseline controller. If additional sensitivity to frequencies near the first bending mode is less critical, the first bending mode band can be notched from the spectral damper input filter. This will negate the decrease in adaptive gain resulting from active damping of the first lateral mode, which may be significant at liftoff (due to hold-down release) and in certain gust environments. Since the output of the controller itself is used in the spectral damping mechanism (rather than, for example, a rate gyro signal), susceptibility to spurious adaptation resulting from noise and flexibility is significantly reduced. This is due to the low-pass characteristic of the linear controller.

\footnotetext{
${ }^{\S}$ The high-pass digital filter ultimately exhibits a band-pass characteristic since the input (the controller output command) has been filtered by the low-pass linear controller.
} 


\section{Leakage}

The leakage term in (1) is proportional to the adaptive gain $k_{a}$ and is determined from the total loop gain $k_{T}$ such that the net effect of (1) always tends toward unity. That is, in the absence of an external stimulus, the adaptive law seeks a gain of $k_{T}=1$ which corresponds to the baseline controller design.

\section{Computational Complexity, Stability Analysis, and Flight Certification}

As a first-order system with simple nonlinearities, the adaptive law can be easily analyzed and exhibits predictable behavior. The adaptation law is implemented in discrete time using forward Euler integration. Three additional states are required to propagate the adaptive law and reference model for each axis, as well as several states for the digital filters associated with the spectral damper. The filters (3) in the digital implementation are are designed directly in the $z$-domain and implemented in a state-space or transfer function form. The use of a simple, low-order reference model also minimizes the computational complexity; no flight software facilities or mathematical operations are required that are not considered "standard" autopilot functions.

The nonlinear feedback element in the present design is highly restricted. While the total loop gain is ultimately dependent on the system states, the architecture decouples the basic feedback control action from the nonlinear portion of the controller. The structure is simple and still relies on classical stability analysis for determination of the limit parameters, and the adaptive controller operation can be readily understood a posteriori by examining the effect of the changing loop gain on the linear, time-invariant approximation of the open-loop plant dynamics. The bounded nature of the adaptation dynamics is easily proved using simple methods for first-order nonlinear differential equations, and these hard limits can be chosen so as to always lie within classical stability margin boundaries for the nominal system. In effect, while the performance potential of the adaptive system would be limited, some classical stability margin could be "allocated" to the adaptive controller to aid in flight certification for initial test and development activities.

In the following section, several test cases are detailed that provide opportunities for adaptive control to demonstrate its adherence to the classical design for the nominal ensemble of vehicles, but also enhance performance or prevent a loss of vehicle (LoV) event. Simulation results employing the present design are presented and discussed.

\section{Simulation Results}

The controller was tested using a high-fidelity time-varying planar model of a typical launch vehicle's pitch axis dynamics in first stage flight. The vehicle dynamics are derived from a linear perturbation model ${ }^{5}$ that includes rigid body aerodynamics, elasticity, actuator dynamics, servoelastic coupling, and sloshing propellant.

Various configurable environmental disturbances were also modeled, including dynamic thrust vector ${ }^{\text {Il }}$ (DTV) errors and a $3 \sigma$ wind shear extracted from a Global Reference Atmosphere Model (GRAM) wind profile. An average of sixteen elastic modes are propagated in the simulation; energy-constrained least squares initialization ${ }^{9}$ of the elastic models is provided at approximately ten second intervals. Unless stated otherwise, all simulation cases include stochastic excitation of the vehicle states due to atmospheric turbulence $(20 \mathrm{ft} / \mathrm{sec} ; 1 \sigma)$ and dynamic motion of the solid rocket motor thrust vector $(0.029 \mathrm{deg} ; 1 \sigma)$.

The baseline controller is a simple proportional-derivative (PD) feedback scheme and uses a rate gyro filter to provide robust phase stabilization of the first lateral bending mode. The solid rocket motor total gimbal angle command is limited in the flight controller to a value of 4 degrees; the gimbal command rate is also limited to 4 degrees per second.

The adaptive control gains are fixed and are identical for all test cases; that is, the adaptive controller has not been tuned to accommodate a particular mode of failure. Vehicle dispersions and potential failure scenarios are not assumed to be known prior to flight. The parameters have been been empirically chosen based on analysis of the openloop linear time-invariant plant frequency response characteristics. In addition, the reference model parameters are determined from analysis of the rigid-body dynamics of the nominal plant. The spectral damper filter characteristics are as shown in Figure 6, and the adaptive controller parameters are given in Table 1 below.

\footnotetext{
"Various uncertainties arise in the actual thrust vector location for large solid rocket motors, including time-varying nozzle gas flow characteristics and the geometric error induced by case depressurization prior to booster separation.
} 


\begin{tabular}{|c|c|c|}
\hline Parameter & Description & Value \\
\hline \hline$a$ & Adaptive error gain & $5 \times 10^{2}$ \\
\hline$\alpha$ & Spectral damping gain & $5 \times 10^{4}$ \\
\hline$\beta$ & Adaptive leakage gain & 0.05 \\
\hline$c$ & Reference error mixing constant & 0.5 \\
\hline$k_{0}$ & Controller baseline gain component (minimum gain) & 0.5 \\
\hline$k_{\max }$ & Maximum adaptive gain & 1.5 \\
\hline$\omega_{r 0}$ & Reference model natural frequency at liftoff (linearly interpolated) & $0.141 \mathrm{~Hz}$ \\
\hline$\omega_{r 1}$ & Reference model natural frequency at separation & $0.173 \mathrm{~Hz}$ \\
\hline$\zeta_{r}$ & Reference model damping ratio & 0.8 \\
\hline
\end{tabular}

Table 1. Adaptive controller parameters

Test cases were selected to demonstrate the baseline and adaptive controller performance within each of the regions considered in the design philosophy described in Figure 1: (1) minimal adaptation, (2) improve performance, and (3) prevent or delay loss of vehicle (LOV). For the nominal vehicle and a benign environment, it is desirable to minimize the impact of augmentation on the total loop gain. As the dispersions increase in severity, improvement in performance and robustness is desirable. Finally, large dispersions leading to vehicle failures should demonstrate the effectiveness of the augmentation beyond the capability of the baseline control system. A summary of the test cases is provided in Table 2, and a subset thereof has been selected for a more detailed discussion.

Many of the following test cases represent a significantly unlikely but credible failure resulting from modeling or design errors in the integrated GN\&C process. These cases are intended to capture the ability of the adaptive controller to recover from scenarios that would be intractable for the baseline controller. As would be anticipated, there also exist many credible scenarios within the capability of the baseline controller in which the vehicle's ability to achieve performance targets is compromised or severely impacted. These scenarios are usually identified via extensive Monte Carlo analysis ${ }^{10}$. Based on the present results, significant improvements in the performance envelope can be expected with the adaptive controller as well. 


\begin{tabular}{|c|c|c|c|}
\hline & Test Case & Category & ObServed Result \\
\hline 1. & Nominal plant and environment & $\begin{array}{l}\text { Minimal } \\
\text { adaptation }\end{array}$ & $\begin{array}{l}\text { Total loop gain remains close to unity; slight decrease in rate of } \\
\text { gimbal command. }\end{array}$ \\
\hline 2. & $\begin{array}{l}\text { LOX \& LH2 slosh damping low and slosh } \\
\text { mass high during first stage flight }\end{array}$ & Minimal adaptation & $\begin{array}{l}\text { First stage slosh does not exhibit sufficient instability to impact } \\
\text { overall system behavior in first stage flight; little adaptation is } \\
\text { observed. }\end{array}$ \\
\hline 3. & $\begin{array}{l}\text { MMH \& NTO2 slosh damping low and slosh } \\
\text { mass high during first stage flight }\end{array}$ & Minimal adaptation & $\begin{array}{l}\text { Payload slosh does not exhibit sufficient instability to impact } \\
\text { overall system behavior in first stage flight; little adaptation is } \\
\text { observed. }\end{array}$ \\
\hline 4. & DTV transient at the end of first stage flight & $\begin{array}{l}\text { Improve } \\
\text { performance }\end{array}$ & $\begin{array}{l}\text { Adaptive augmentation provides a slight performance increase } \\
\text { during tailoff. No significant changes to attitude and rate errors at } \\
\text { separation. Responsiveness improved; increase in gimbal margin. }\end{array}$ \\
\hline 5. & $\begin{array}{l}\text { High inertia / low thrust with wind } \\
\text { disturbance }\end{array}$ & $\begin{array}{l}\text { Improve } \\
\text { performance }\end{array}$ & $\begin{array}{l}\text { Slight performance improvement, especially during pitch-over } \\
\text { maneuver. }\end{array}$ \\
\hline 6. & $\begin{array}{l}\text { Low performance due to error in loaded gain } \\
\text { table data; wind disturbance }\end{array}$ & $\begin{array}{l}\text { Improve } \\
\text { performance }\end{array}$ & $\begin{array}{l}\text { Stability maintained using baseline controller but performance is } \\
\text { impacted. Adaptive controller provides a slight performance } \\
\text { improvement. }\end{array}$ \\
\hline 7. & Low inertia / high thrust & Prevent LoV & $\begin{array}{l}\text { Baseline controller gain is excessive, resulting in loss of vehicle } \\
\text { control. Adaptive augmentation responds to excitation of the rigid } \\
\text { body dynamics and reduces loop gain to maintain stability. }\end{array}$ \\
\hline 8. & $\begin{array}{l}\text { Controller gain excessive due to } \\
\text { gain-loading error }\end{array}$ & Prevent LoV & $\begin{array}{l}\text { Baseline controller induces structural instability. Adaptive } \\
\text { augmentation responds to structural resonance and reduces loop } \\
\text { gain to maintain stability. }\end{array}$ \\
\hline 9. & $\begin{array}{l}\text { Excessive static aerodynamic instability; } \\
3 \sigma \text { wind shear environment }\end{array}$ & Prevent LoV & $\begin{array}{l}\text { Baseline controller unable to maintain aerodynamic stability } \\
\text { when excited by wind shear, resulting in loss of vehicle. } \\
\text { Adaptive controller maintains aerodynamic stability. }\end{array}$ \\
\hline 10. & $\begin{array}{l}\text { First bending mode exhibits significant } \\
\text { deviation in anticipated frequency and } \\
\text { modal gain at sensor }\end{array}$ & Prevent LoV & $\begin{array}{l}\text { Baseline controller excites first bending mode to resonance, } \\
\text { resulting in probable structural failure. Adaptive controller } \\
\text { detects resonance and reduces gain. }\end{array}$ \\
\hline
\end{tabular}

Table 2. Summary of test cases (bold denotes tests to be described in more detail) 
In all simulation cases, the baseline controller acts without augmentation until 10 seconds after liftoff. This provides sufficient time to execute the flyaway, damp liftoff structural transients, and allow the vehicle to clear the tower. Azimuth alignment and pitchover maneuvers have already begun when adaptation is enabled. The adaptation remains active throughout the remainder of the first stage flight. The spectral damping filter, however, is initialized at liftoff and remains online throughout the entire flight in order minimize filter transients.

The simulation is propagated at an integration rate of $200 \mathrm{~Hz}$ using a fourth order Runge-Kutta (RK4) algorithm. The baseline and adaptive control algorithms, including the digital filters and reference model dynamics, are computed in discrete time at $50 \mathrm{~Hz}$. The simulation is continued until just before the nominal booster separation event. The simulation is stopped prematurely in the case of a vehicle load exceedance, which is assumed to occur for the purposes of the simulation at a rigid-body load indicator $(\bar{q} \alpha)$ value of $8000 \mathrm{psf}$-deg or an instantaneous elastic strain energy of $1 \times 10^{4} \mathrm{ft}-\mathrm{lbf}$, depending on the test case. These limits are chosen empirically and are a conservative approximation to identify cases with high structural loads. The latter value is highly dependent on the number and type of modes in the truncated elastic model.

\section{A. Nominal Plant \& Environment - Minimal Adaptation (Case 1)}

For this test case, the nominal plant (including flexibility, slosh, and aerodynamics) is simulated. In this scenario, the baseline controller is expected to have reasonable performance. Thus, it is desirable for the contribution from the adaptive augmentation to be minimal so that the vehicle retains near-nominal performance.

The pitch angle and rate exhibit negligible variation due to the presence of the adaptive augmentation (Figure 7). Some adaptation is visible during the pitch-over maneuver when the rigid body mode is excited. The abrupt change in the rate of the adaptation during this maneuver occurs when the augmentation is activated at $t=10 \mathrm{sec}$. This causes a small decrease in the adaptive gain which allows the vehicle to maintain similar performance but provides a slight decrease in the rate of the gimbal command (Figure 7). The total gain $k_{T}$ is attracted to unity as the flight continues, as designed. The results of this test demonstrate that the contribution from the adaptive augmentation is minimal when it is not needed; adaptation does not negatively impact the vehicle's performance.
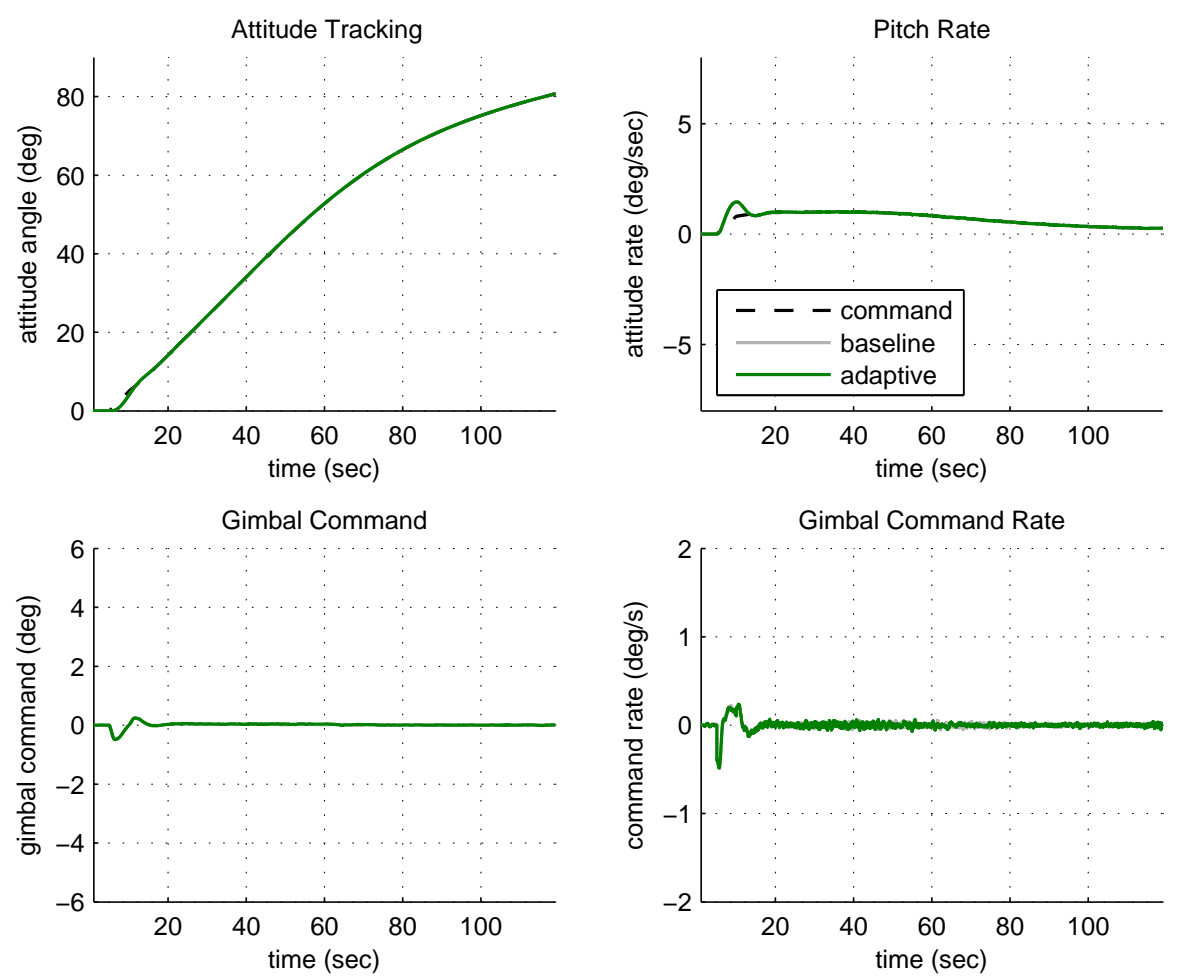

Figure 7. Nominal plant: Minimal deviations in attitude and rate with a slight decrease in the rate of the gimbal command. 
AC Total Adapted Gain
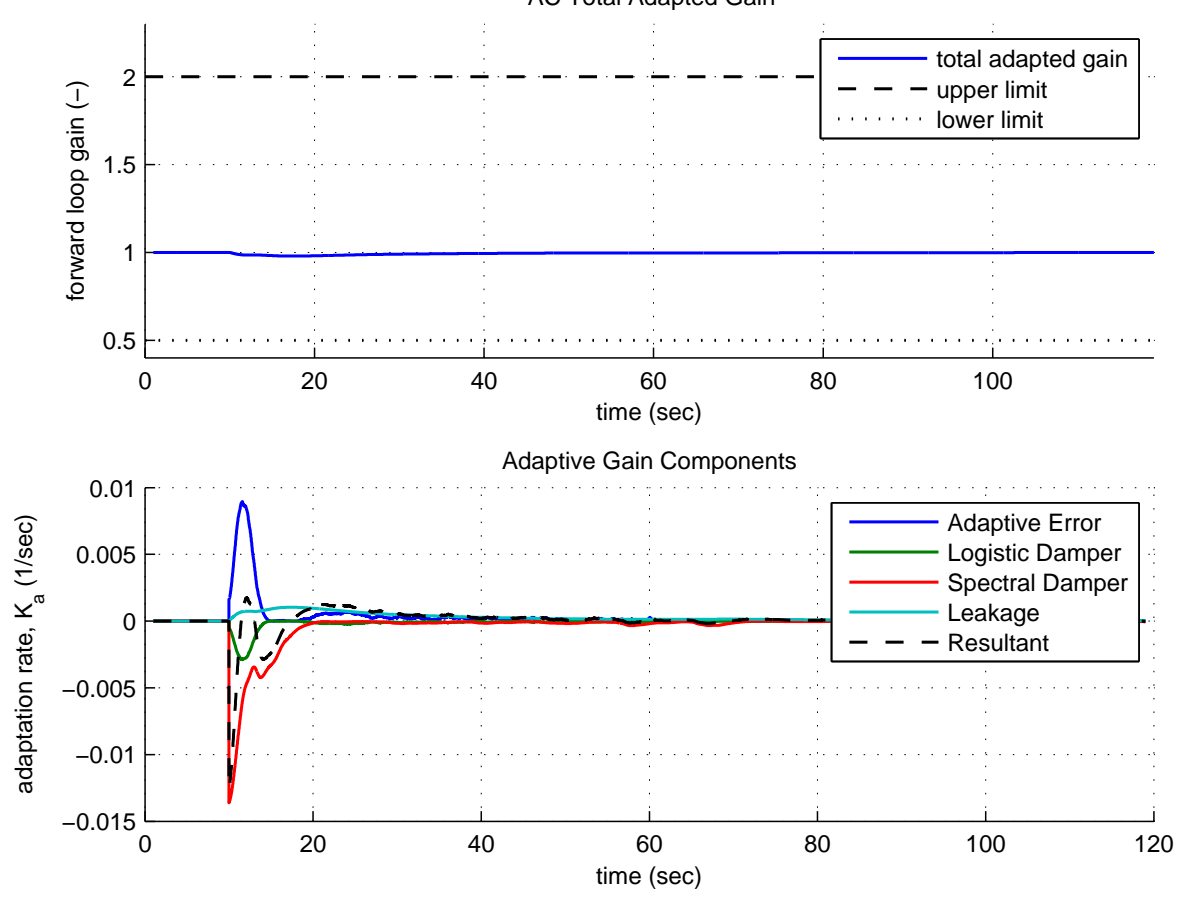

Figure 8. Nominal plant: Loop gain and with adaptation stays near unity.

\section{B. Large Aerodynamic Moment - Prevent Loss of Vehicle (Case 9)}

A large aerodynamic modeling error is simulated by tripling the aerodynamic moment coefficient in the pitch axis. In addition, the vehicle experiences a large wind shear near maximum dynamic pressure $(t=60 \mathrm{sec})$ that necessitates a rapid slew of the thrust vector in order to maintain stability. In the absence of adaptive augmentation, these conditions drive the vehicle rapidly towards aerodynamic divergence and its structural load limit (Figures 9 and 10). With adaptive augmentation, the loop gain increases as the error between the attitude and the expected attitude (output of the reference model) accumulates (Figure 11).

Each element contributing to the rate of adaptation is shown in Figure 11. Note that the spectral damper signal remains small, indicating that the rigid body and flexible modes are not being excited, and therefore this component does not hinder the increase in loop gain. The logistic term damps the continued increase in adaptation as the magnitude of the gain grows, which can be observed as the total loop gain approaches $k_{T}=1.5$ near maximum dynamic pressure.

Both the baseline and adaptive frequency response near the point of divergence are shown in Figure 12. While the nominal aerodynamic gain margin decreases to zero and then becomes unstable, the increase in effective loop gain due to the augmentation is able to retain stability in the linear time-invariant sense. 

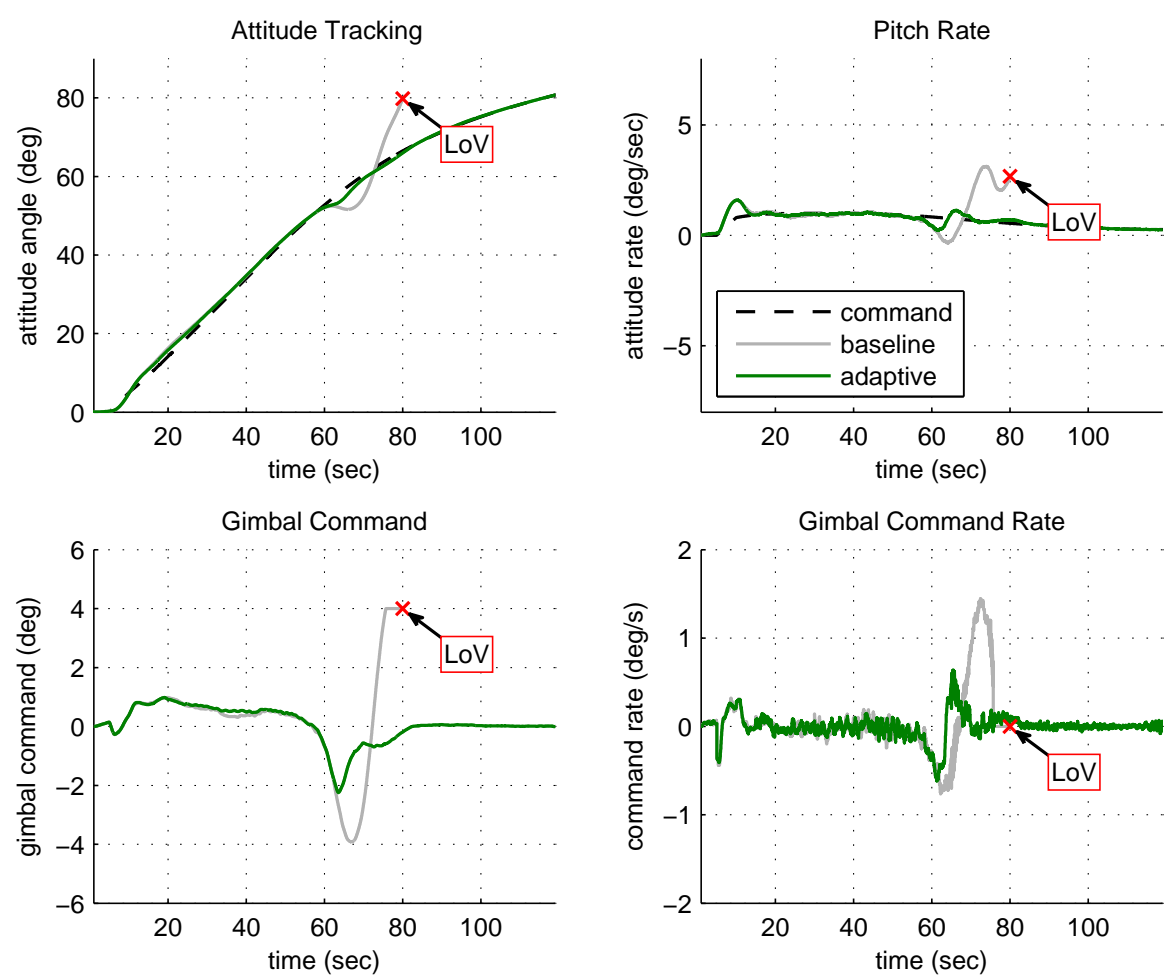

Figure 9. Large aerodynamic moment: tracking performance \& control command

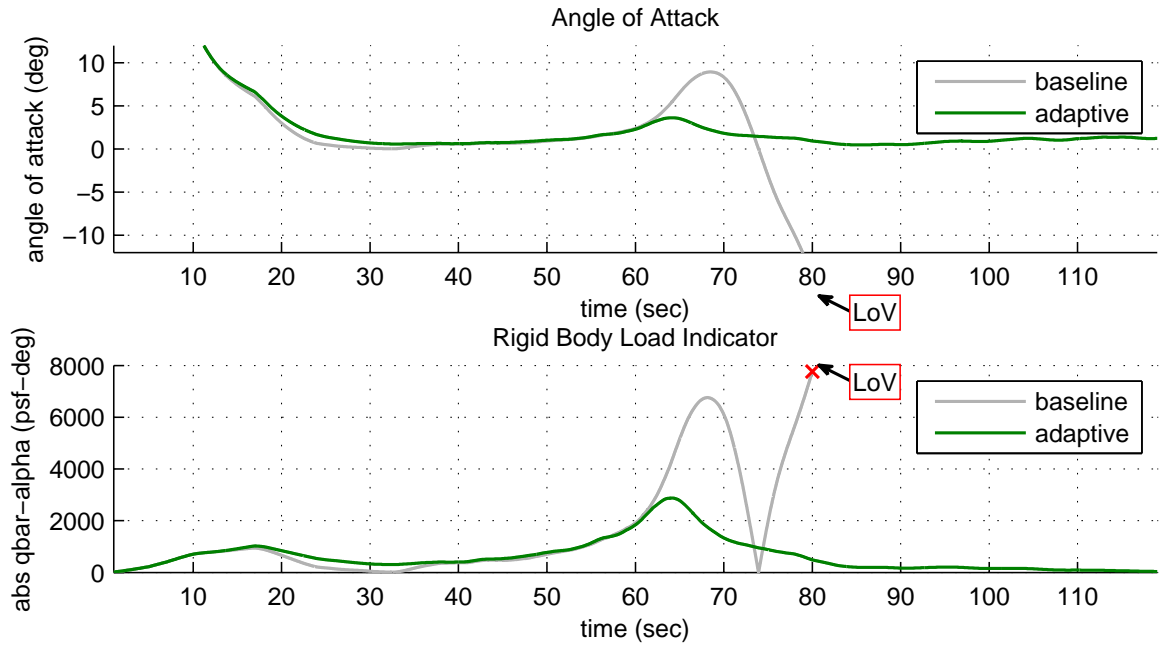

Figure 10. Large aerodynamic moment: rigid body load indicator 

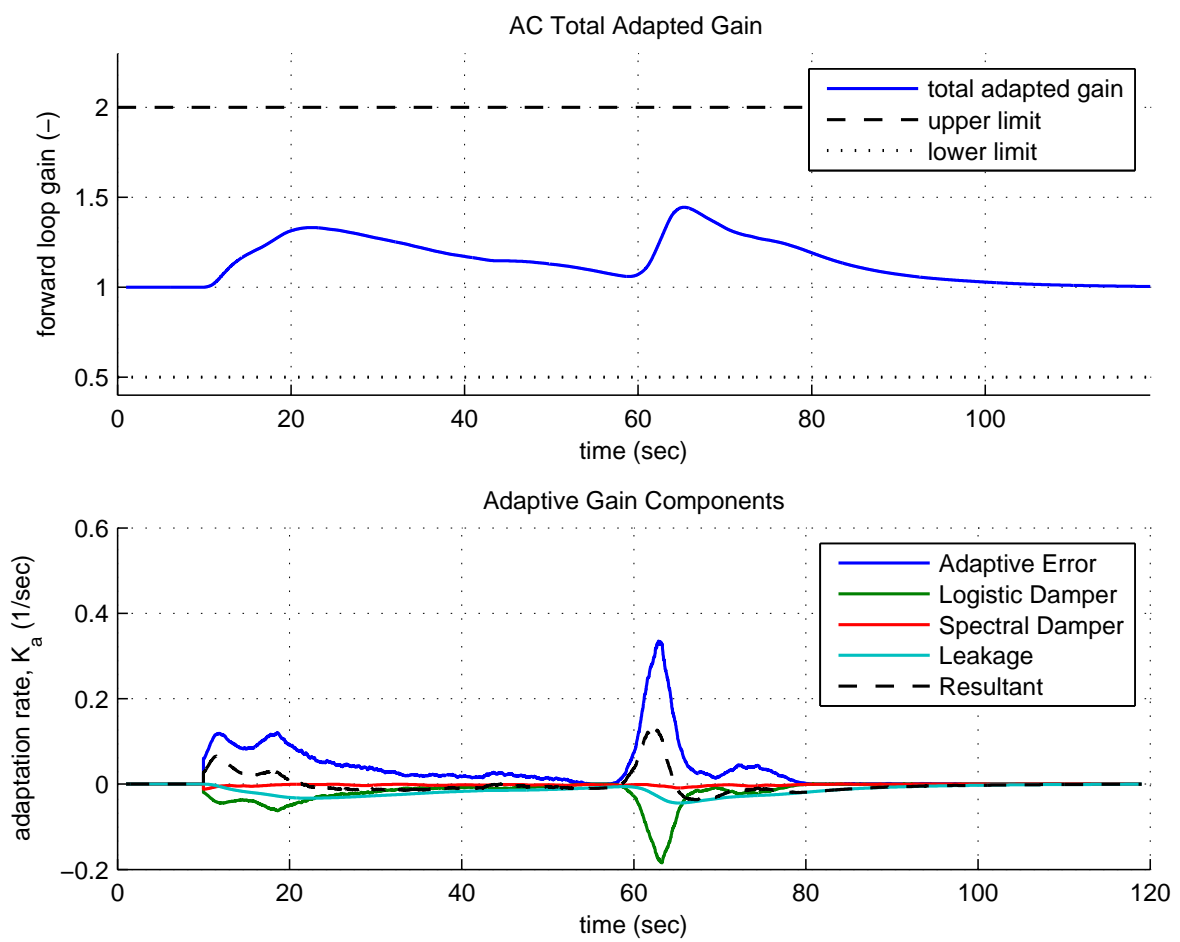

Figure 11. Large aerodynamic moment: adaptive gain

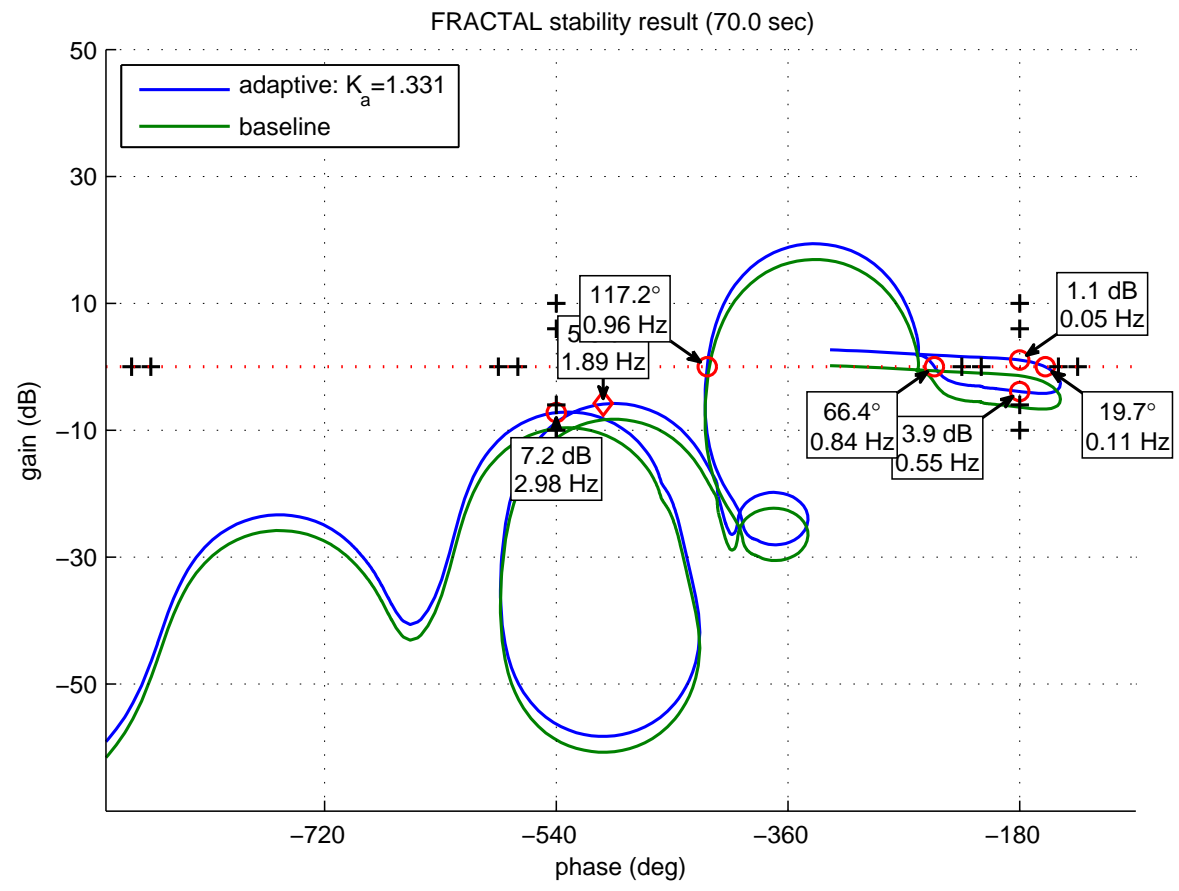

Figure 12. Large aerodynamic moment: frequency domain 


\section{First Bending Mode Unstable - Prevent Loss of Vehicle (Case 10)}

In this test case, the first lateral bending mode frequency is reduced by $30 \%$ and the modal gain at the sensor is increased by $6 \mathrm{~dB}$. Although variations in the frequency of the first lateral bending mode from the modeled value often fall within $5-10 \%$ based on test data ${ }^{11}$, this scenario represents the possibility of a modeling error not resolved prior to flight by an integrated vehicle ground vibration test (IVGVT).

The modification to the first flexible mode is outside the robustness envelope of the baseline controller and drives the system unstable (Figure 13). The elastic instability is also apparent based on the nominal open-loop frequencydomain analysis, an example of which is shown in Figure 16 at 20 seconds flight time. The commanded gimbal response will be predominantly in-phase with the elastic vehicle motion, exciting the structure at increasing amplitudes until it reaches its load limit (Figure 13).

The augmenting controller response is dominated by the signal captured through the spectral damping filter (Figure 14). The undesirable vehicle motion increases as the vehicle begins its pitch-over maneuver. Adaptation drives the adaptive loop gain down near its lower bound of 0.5 as soon as the adaptive controller is enabled 10 seconds after liftoff. The vehicle retains stability throughout the entirety of first stage flight. Leakage and error-driven adaptation attract the loop gain back to unity as the flight continues, allowing the vehicle to re-gain performance as the bending mode shifts up in frequency.
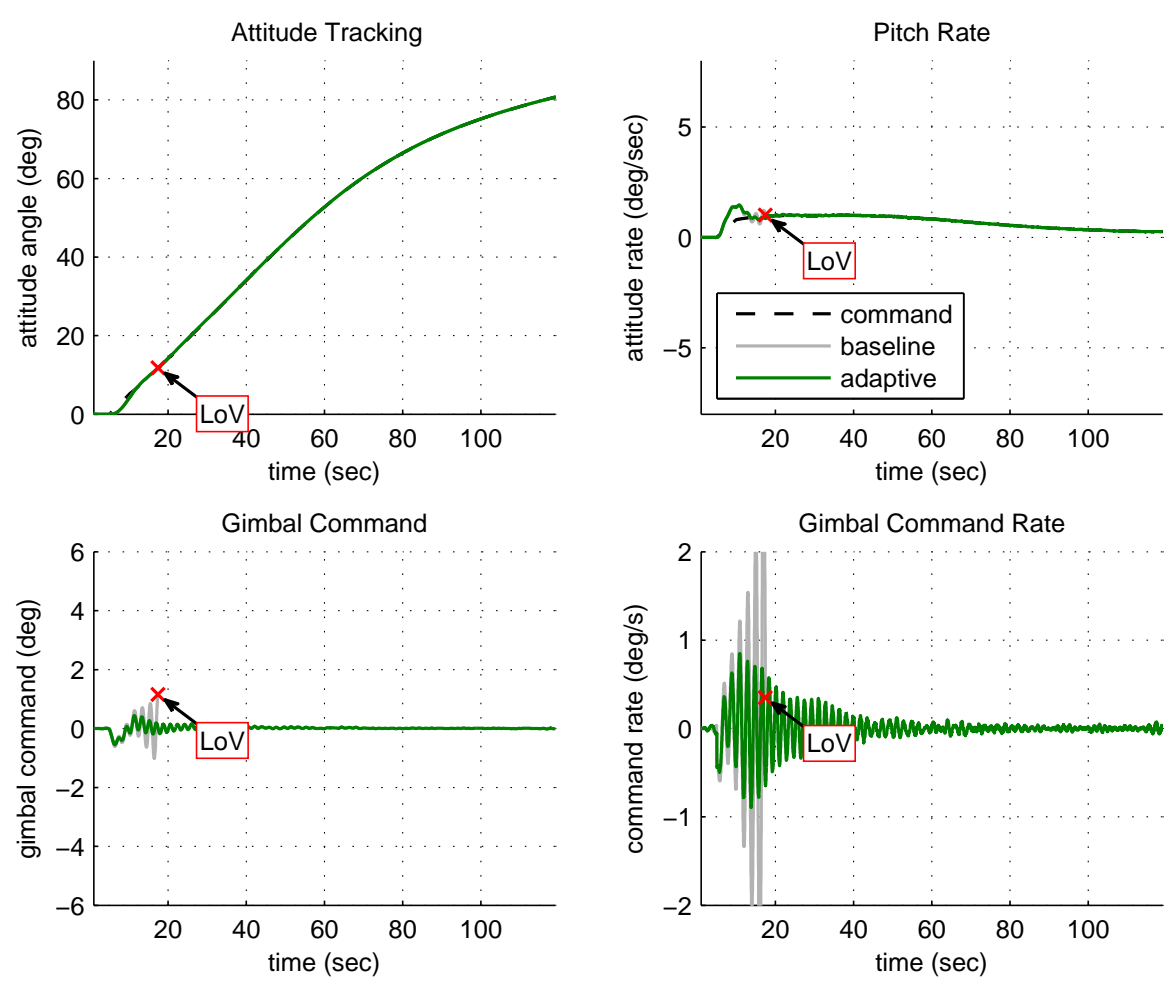

Figure 13. Large variation in first bending mode: tracking performance $\&$ control command 


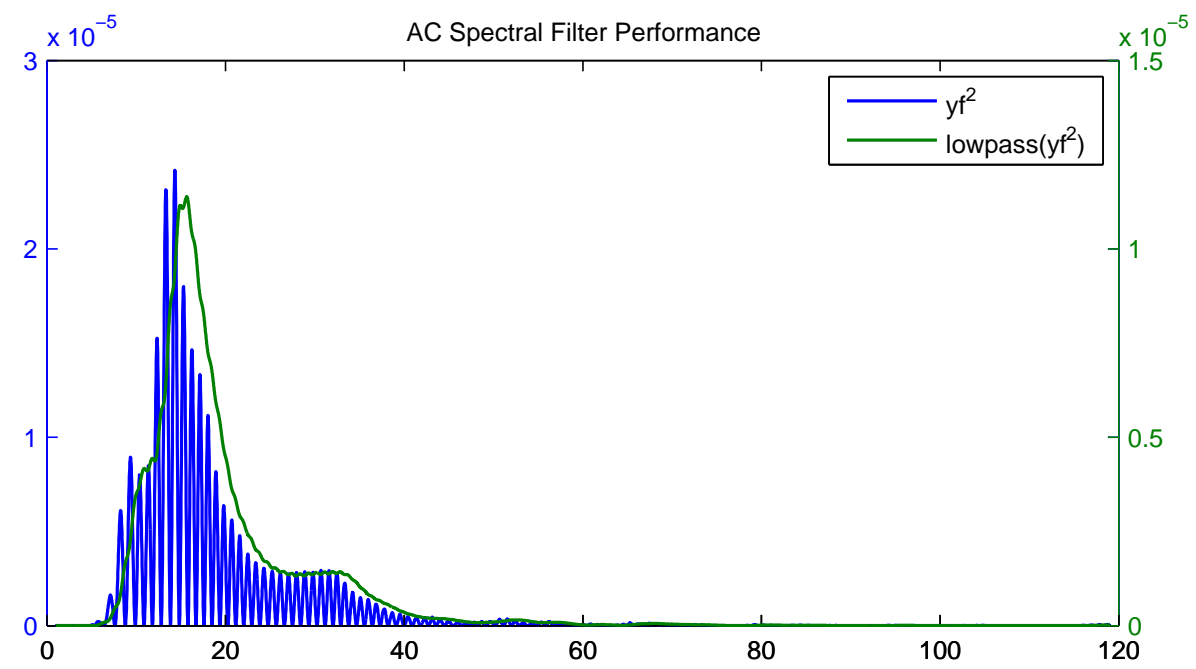

Figure 14. Large variation in first bending mode: spectral damping filter
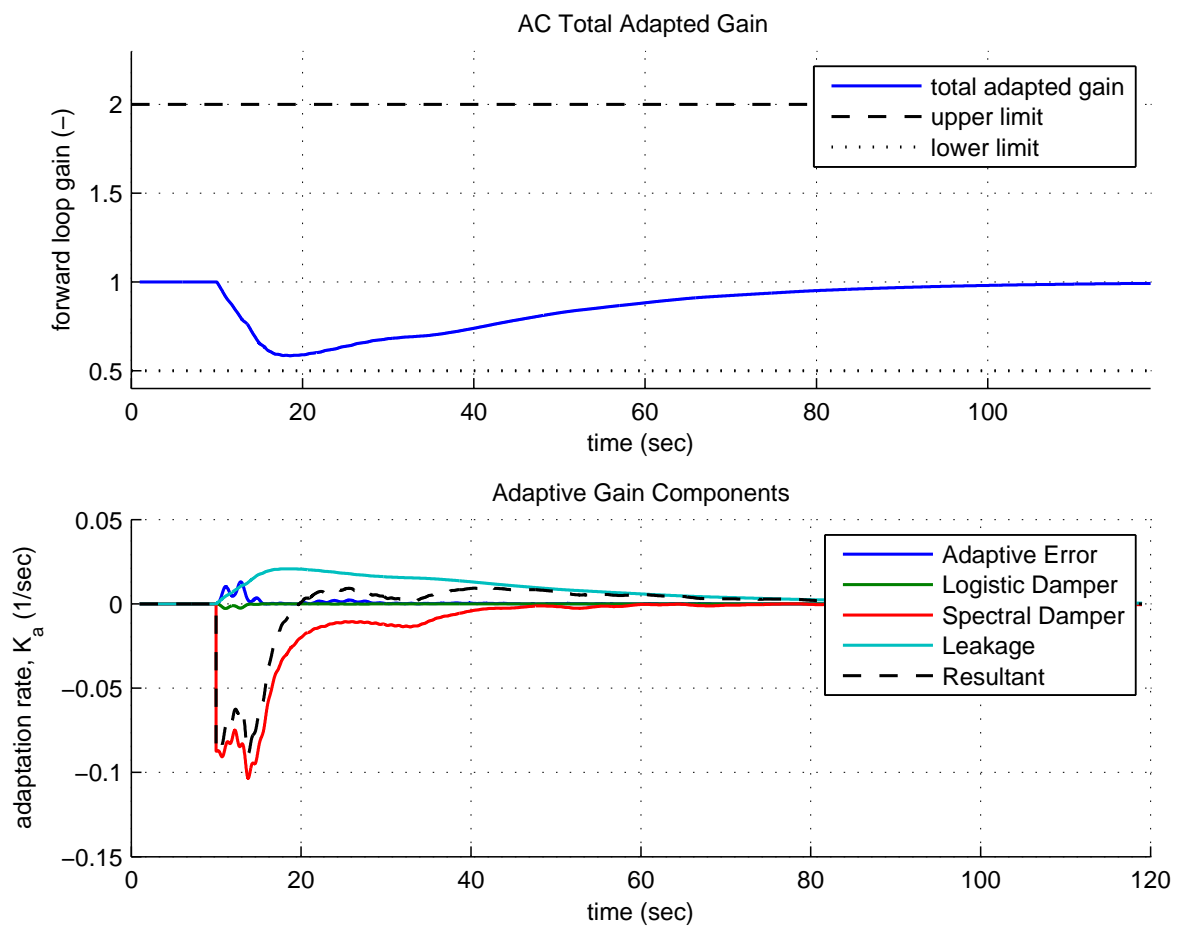

Figure 15. Large variation in first bending mode: adaptive gain 


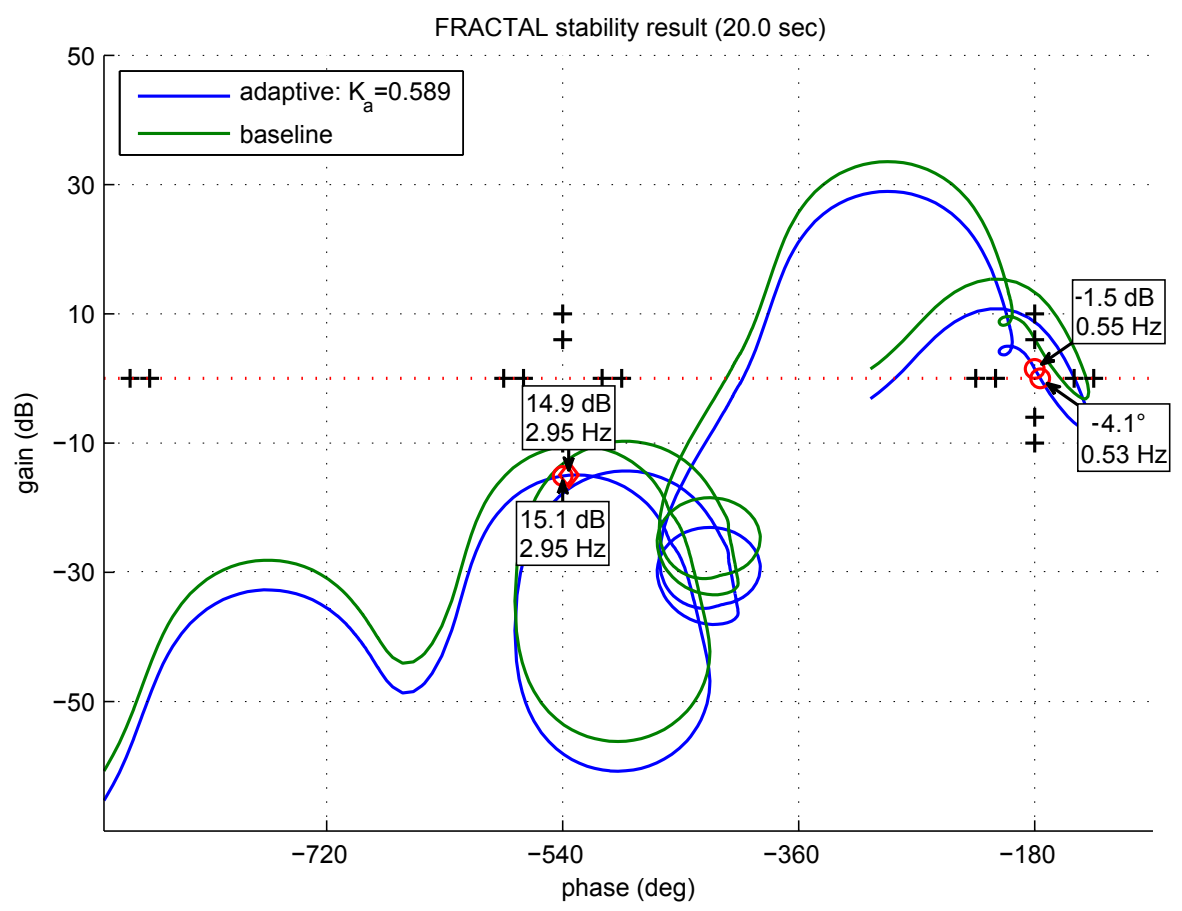

Figure 16. Large variation in first bending mode: frequency response at liftoff 


\section{Conclusions and Forward Work}

A practical, adaptive augmenting control (AAC) algorithm has been developed specifically for the launch vehicle application, taking into account its dynamic features and industry standards for assessing stability. The design targets an augmentation of a well-tuned classically designed baseline controller. It features a multiplicative loop gain that increases only for off-nominal conditions when performance enhancements are needed, an ability to reduce this gain in order to mitigate control-structure interaction, and automatic re-convergence to the baseline controller design. Additional mechanisms are included to constrain the multiplicative signal in accordance with classical margin requirements so as to further mitigate risk. The algorithm was tested using a high-fidelity simulation of a typical large launch vehicle with the usual PD-type baseline flight control system.

Ten test cases were considered to verify that the augmentation deviates minimally from the baseline controller for near-nominal vehicle and environmental conditions but is capable of improving performance and preventing a loss of vehicle (LOV) for more aggressive dispersions. Results verified that the augmentation performed as expected for cases ranging from a nominal flight to significant variations in to the modeled aerodynamic and elastic characteristics.

Forward work will require a thorough evaluation of the proposed algorithm in a full six-degree-of-freedom nonlinear simulation environment that includes the higher-order, nonlinear effects of sensor dynamics, noise, and closed-loop guidance. Monte Carlo analysis will be performed to ascertain the ability of the present design to improve performance capability in a statistical sense. In addition, the architecture and method of error computation must be modified slightly to integrate with anti-drift/load-relief (AD/LR) flight control algorithms, whose application can be expected in future launch vehicle development programs.

Finally, it is anticipated that the present design may also find application in the reduction of range dispersions on guided sounding rocket platforms, where the control effectiveness is also highly uncertain due to wide-ranging variations in the thrust, mass profile, and atmospheric properties. This application represents an attractive test opportunity to validate the proposed algorithm in a relevant flight environment. 


\section{References}

${ }^{1} \mathrm{~J}$. Blakelock, Stability and Control of Aircraft and Missiles, 2nd Ed. Wiley-Interscience, 1991.

${ }^{2}$ M. Thompson and J. Hunley, Flight Research: Problems Encountered and What They Should Teach Us. NASA SP-2000-4522, 2000. 1967.

${ }^{3}$ A. Greensite, "Analysis and Design of Space Vehicle Flight Control Systems, Volume I - Short Period Dynamics," tech. rep., NASA CR-820,

${ }^{4}$ J. Frosch and D. Vallely, "Saturn AS-501/S-IC Flight Control System Design,” J. Spacecraft, vol. 4, no. 8, pp. 1003-1009, 1967.

${ }^{5}$ J. Orr, M. Johnson, J. Wetherbee, and J. McDuffie, "State Space Implementation of Linear Perturbation Dynamics Equations for Flexible Launch Vehicles," in AIAA Guidance, Navigation, and Control Conference, Chicago, Illinois, AIAA-2009-5962, 2009.

${ }^{6} \mathrm{~J}$. Hanson, "A Plan for Advanced Guidance and Control Technology for 2nd Generation Reusable Launch Vehicles," in AIAA Guidance, Navigation, and Control Conference, Monterey, CA, AIAA-2002-4557, 2002.

${ }^{7}$ M. Thompson and J. Welsh, "Flight Test Experience With Adaptive Control Systems," tech. rep., NASA Flight Research Center, 1970.

${ }^{8}$ Staff of the Flight Research Center, "Experience with the X-15 adaptive flight control system," tech. rep., NASA Flight Research Center, TN D-6208, 1971.

${ }^{9}$ J. Orr, "Elastic Model Transitions Using Quadratic Inequality Constrained Least Squares," tech. rep., NASA Marshall Space Flight Center, Control Systems Design and Analysis Branch (EV41), September 2011.

${ }^{10}$ J. Hanson and C. Hall, "Learning About Ares I from Monte Carlo Simulation," in AIAA Guidance, Navigation, and Control Conference, Honolulu, HI, AIAA-2008-6622, 2008.

${ }^{11}$ N. S. V. D. C. (Structures), “Structural Interaction with Control Systems,” tech. rep., NASA Marshall Space Flight Center, SP-8079, 1971.

\section{Acknowledgments}

This research was supported by the NASA Marshall Space Flight Center under contract NNM06AA01Z. The authors wish to thank our colleagues in the MSFC Flight Mechanics and Analysis Division, especially Steve Ryan, Dave Edwards, and Charles Hall, for their numerous insights and contributions throughout the course of this work. In addition, the authors thank Jimmy Jang of the Charles Stark Draper Laboratory for his constructive feedback regarding multiplicative adaptive systems. 


\section{Robust, Practical Adaptive Control for Launch Vehicles}

Jeb S. Orr

The Charles Stark Draper Laboratory, Inc.

\section{Tannen S. VanZwieten}

NASA Marshall Space Flight Center

Control Systems Design and Analysis Branch (EV41)

2012 AIAA Guidance, Navigation, and Control Conference Minneapolis, Minnesota August 13-16, 2012 


\section{Motivation for Adaptive Augmentation}

- In the absence of vehicle or environmental uncertainty, a fixed-gain controller is optimized prior to flight (no motivation for adaptation)

- Conservatism in launch vehicle design generally yields well-performing classical controllers

- There is no desire to improve on the well-tuned baseline control system design for nominal cases

- Adaptive control provides additional robustness by using sensed data to adjust the gain on-line

\section{AAC Objectives}

- "Do no harm"

- Maintain consistency with classical design approach

- Protect nominal control gains

- Increase robustness; prevent / delay loss of vehicle (LOV)

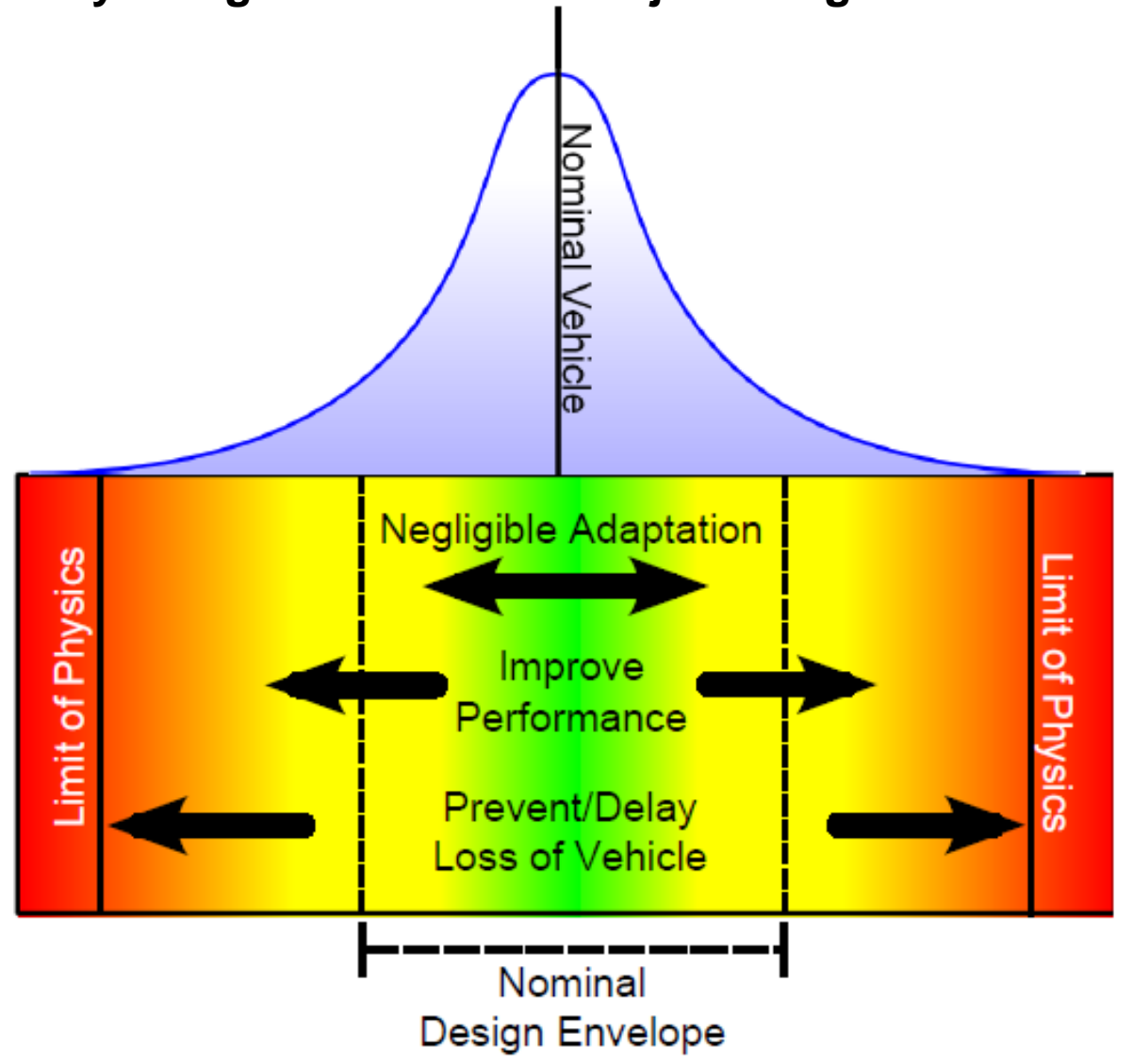


- A new algorithm based on heritage multiplicative SISO adaptive gain concepts developed for NASA heritage flight control systems has been developed for launch vehicle adaptive control

- The present algorithm relies on model reference-driven bounded gain adaptation supplemented by spectral damping

- Upper and lower bounds for gain adaptation can be reconciled in terms of classical LTI frequency-domain stability margins

- The spectral damping mechanism uses frequency-domain feedback to suppress undesirable control-structure interaction

- The adaptation law does not require persistent excitation and seeks the nominal design

- The algorithm is simple, has predictable behavior, and is compatible with characteristics of launch vehicle plants that are conditionally stable in gain

- $1^{\text {st }}$ order scalar ODE with bounded behavior

- Adaptation limits are chosen by the designer based on classical criteria

- Includes an ability to decrease system performance in order to regain stability in the presence of uncertain parasitic dynamics (especially bending and sloshing modes) 


\section{History}

- Current architecture has heritage to flight-tested systems

- [Minneapolis-Honeywell] MH-90 (F-101) and MH-96 (X-20, X-15)

- Based on a prescribed servo limit cycle amplitude (marginal servo poles)

- Saw numerous flight tests (>60) on X-15-3, improved performance and pilot opinion of handling qualities over wide-ranging flight envelope

- Experienced operational problems, including contributing to a LOV

- Caused by difficulties in analog implementation, EMI, and interaction with pilot

- Changes in architecture and design paradigm mitigate all known operational issues

- The exact cause of the failure has been misunderstood and may have led to a reduction in the momentum of adaptive control ca. 1967

- The concept is well-suited to a digital implementation
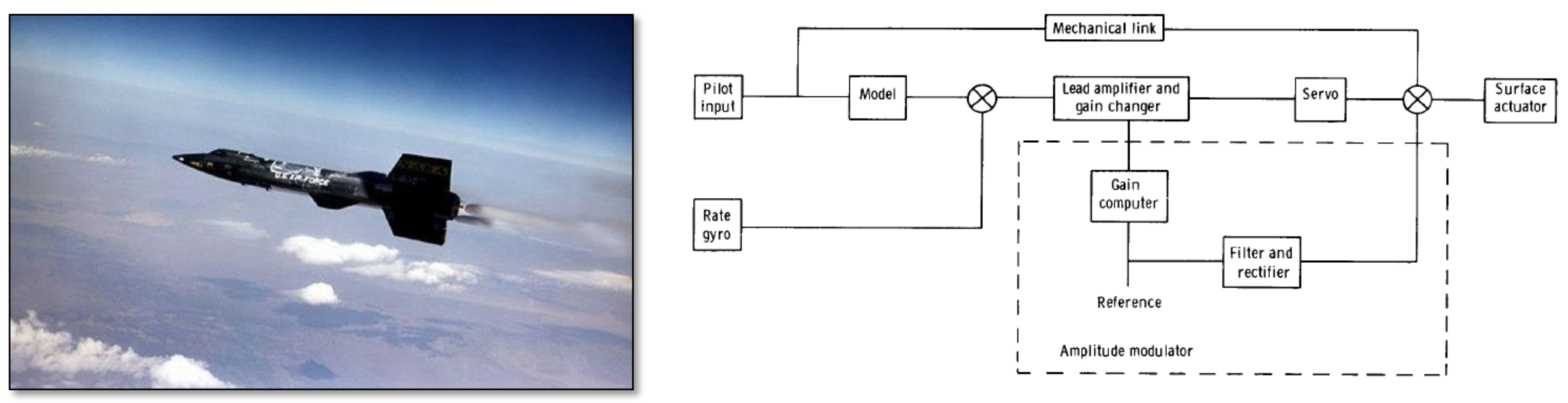


\section{Classical Feedback Control Tradeoffs}

- The performance and stability of a classical feedback control system are traded based on the forward loop gain $k_{T}$

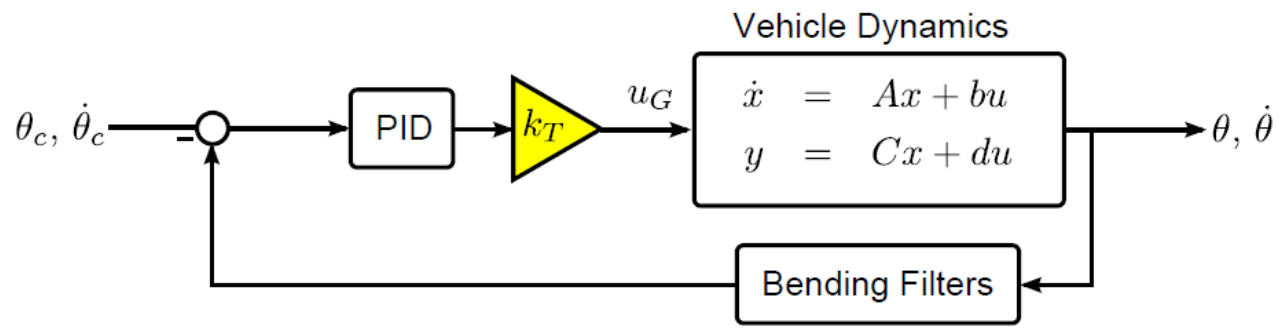

- For flexible vehicles and systems with internal dynamics (e.g. actuators), we are controlling the lowfrequency dynamics (rigid-body) while trying to suppress the high-frequency dynamics (flexibility)

- Systems of this type operate between a minimum gain for performance and a maximum gain for stability (conditional stability)

- We'd like the gain to be as high as possible to improve performance, but we are limited by uncertainty in the high-frequency dynamics

- In a launch vehicle, the controlled error dynamics are lower in frequency than the internal dynamics (flex, slosh) and parasitic (servo) dynamics

- As we approach instability due to insufficient forward loop gain (aerodynamics, thrust uncertainty), the error dynamics diverge exponentially or at a frequency at or below the design control frequency

- As we approach instability due to excessive forward loop gain, we observe the introduction of highfrequency spectrum in the control loop

- These are general characteristics of the control of damped complex roots with servo lag, delay, and complex servo poles 


\section{Conditional Stability}

- Most launch vehicles are conditionally stable with respect to autopilot gain

- If we knew the system parameters exactly, we could design the system gain to be very close to the maximum achievable

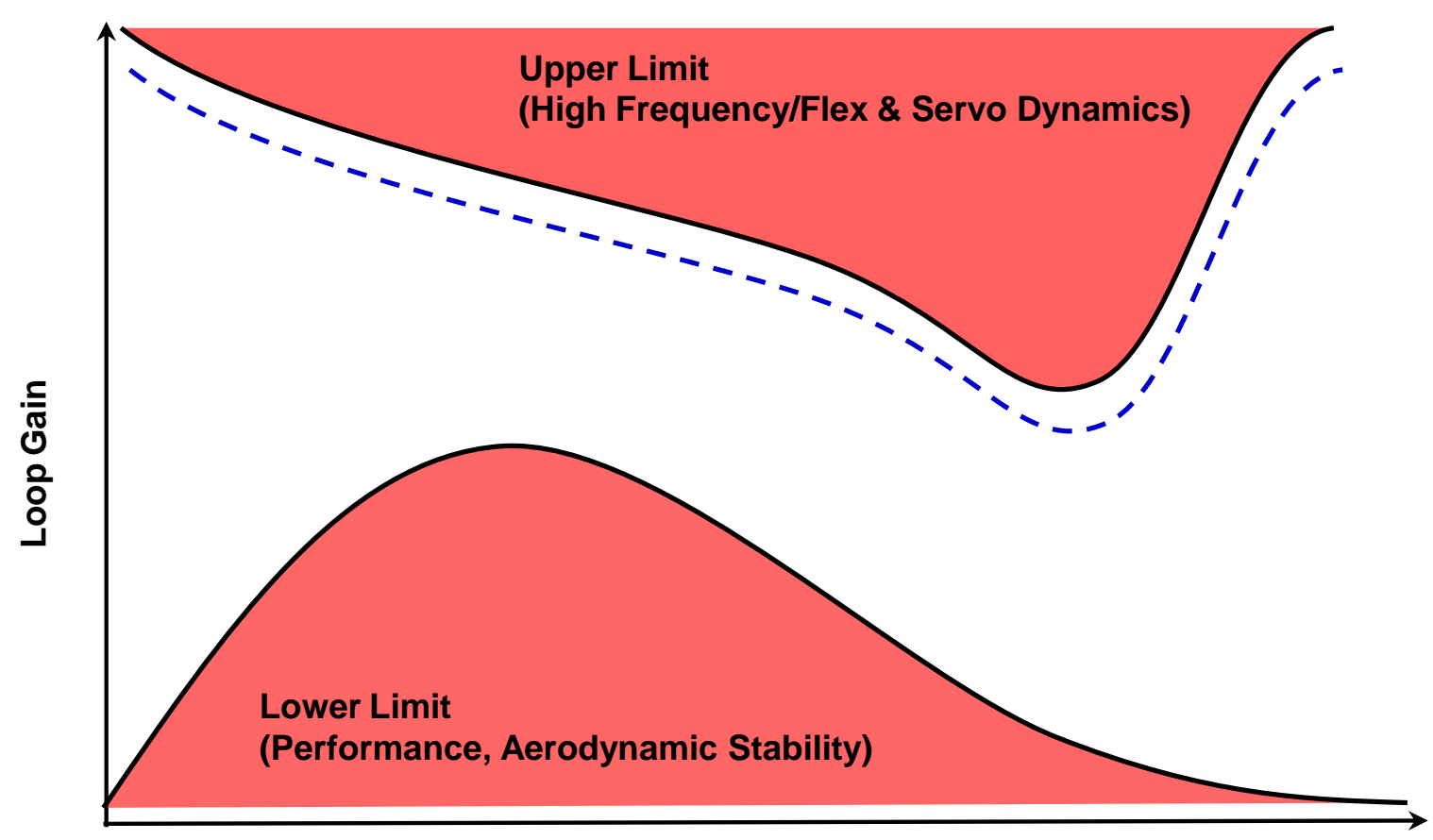

Flight Time 


\section{Conditional Stability (II)}

- Because of uncertainty in the models, we have to design with sufficient gain margins

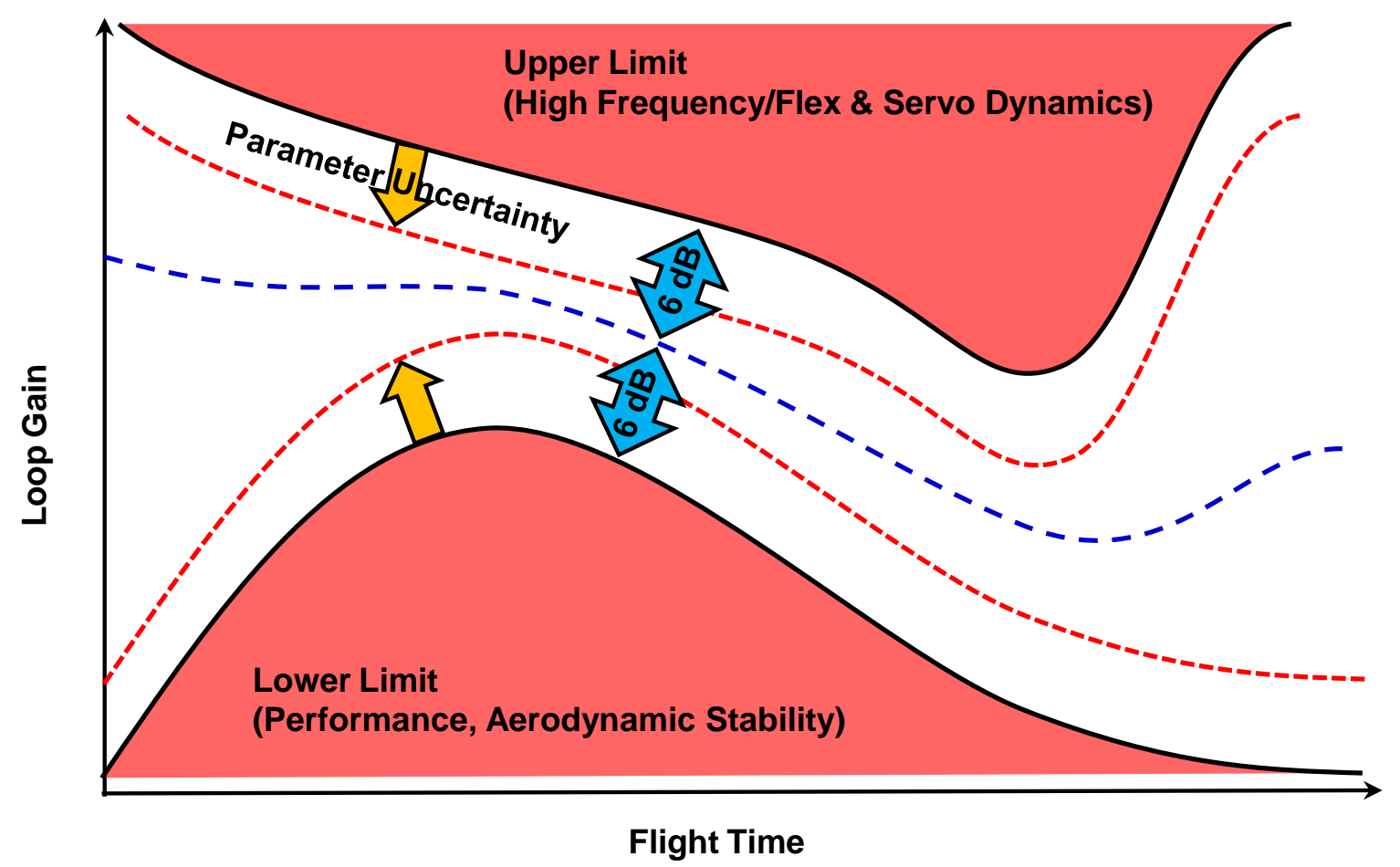

-What if we could sense the upper and lower limits "on-the-fly"? 


\section{Spectral Damping Concept: Example System}

- Consider the $4^{\text {th }}$ order plant dynamics with $2^{\text {nd }}$-order servo

$$
G_{p}(s)=\underbrace{\left(\frac{\omega_{s}^{2}}{s^{2}+2 \zeta \omega_{s} s+\omega_{s}^{2}}\right)}_{\text {servodynamics }} \underbrace{\left(\frac{1}{s^{2}+\kappa}\right)}_{\text {unstable plant }}, \kappa<0
$$

- The plant is open-loop unstable if $\kappa<0$ (negative stiffness, like aerodynamic instability)

- The system can be easily stabilized with proportional-derivative feedback control

$$
C(s)=K_{T}\left(K_{p}+K_{d} s\right)
$$

- Due to servo lag, the rigid body poles or the servo poles are unstable at some positive gain $K_{T}$.

- The system is conditionally stable.

- $K_{T}$ is the controller forward loop gain. With the proportional-derivative control gain ratios fixed, the system performance will improve with increasing gain until we approach instability.

- We design the nominal control system based on knowledge of $\kappa$. The nominal response is acceptable.

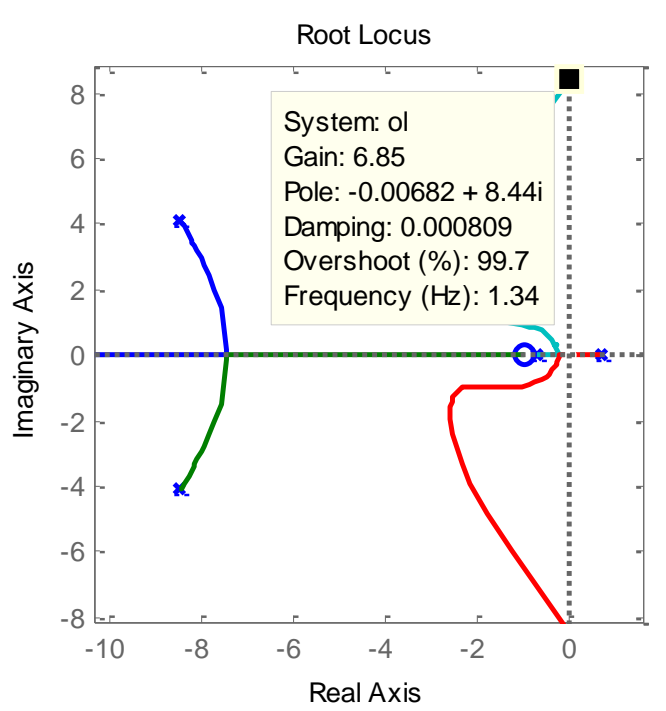




\section{Spectral Damping Concept}
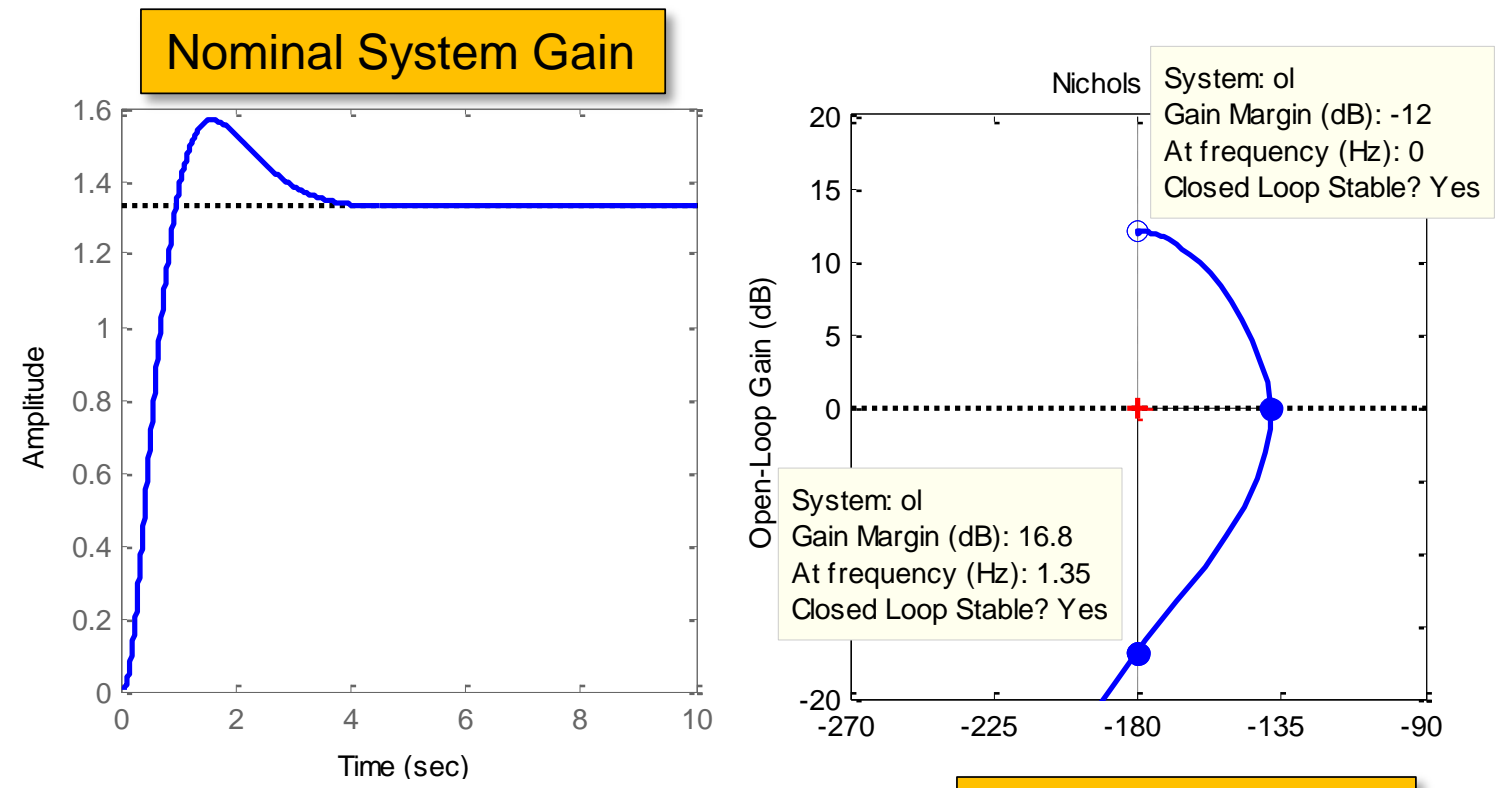

- As the system reaches the stability limit, we see frequency content in the control loop at a higher frequency than the error dynamics to be controlled

- This frequency content is used as a gain adaptation damping mechanism
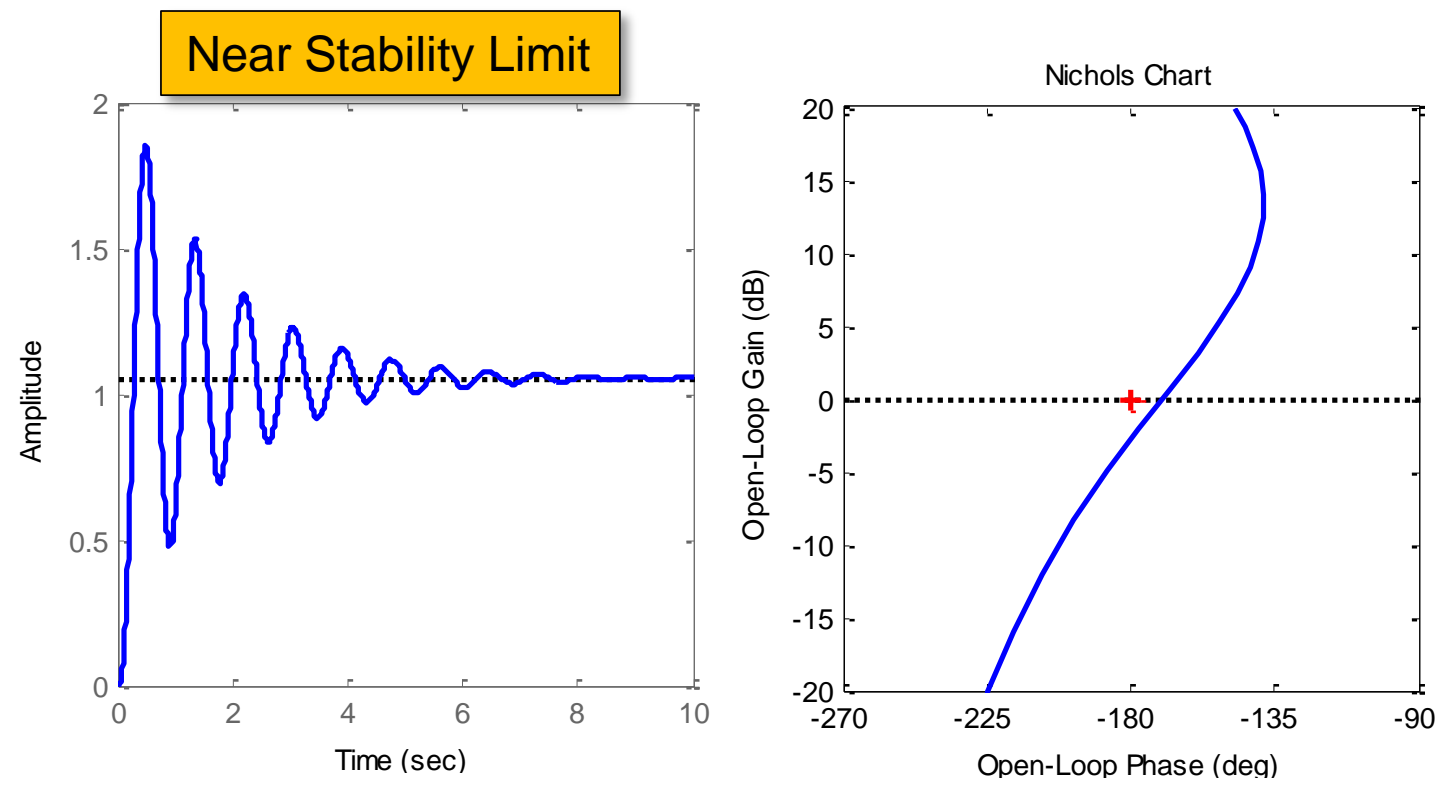


\section{Adaptive Augmenting Design}

\section{- Assume a well-tuned classical controller for the nominal system}

- The forward loop gain $k_{T}$ is augmented by a signal $k_{a}$

- The total gain is formed from a fixed minimum gain and the augmenting gain;

$$
k_{T}=k_{0}+k_{a}
$$

- Multiplicative augmentation is easy to assess in terms of gain margin

- The update law for the augmenting signal depends on the command, sensed attitude and rate, and the baseline controller output

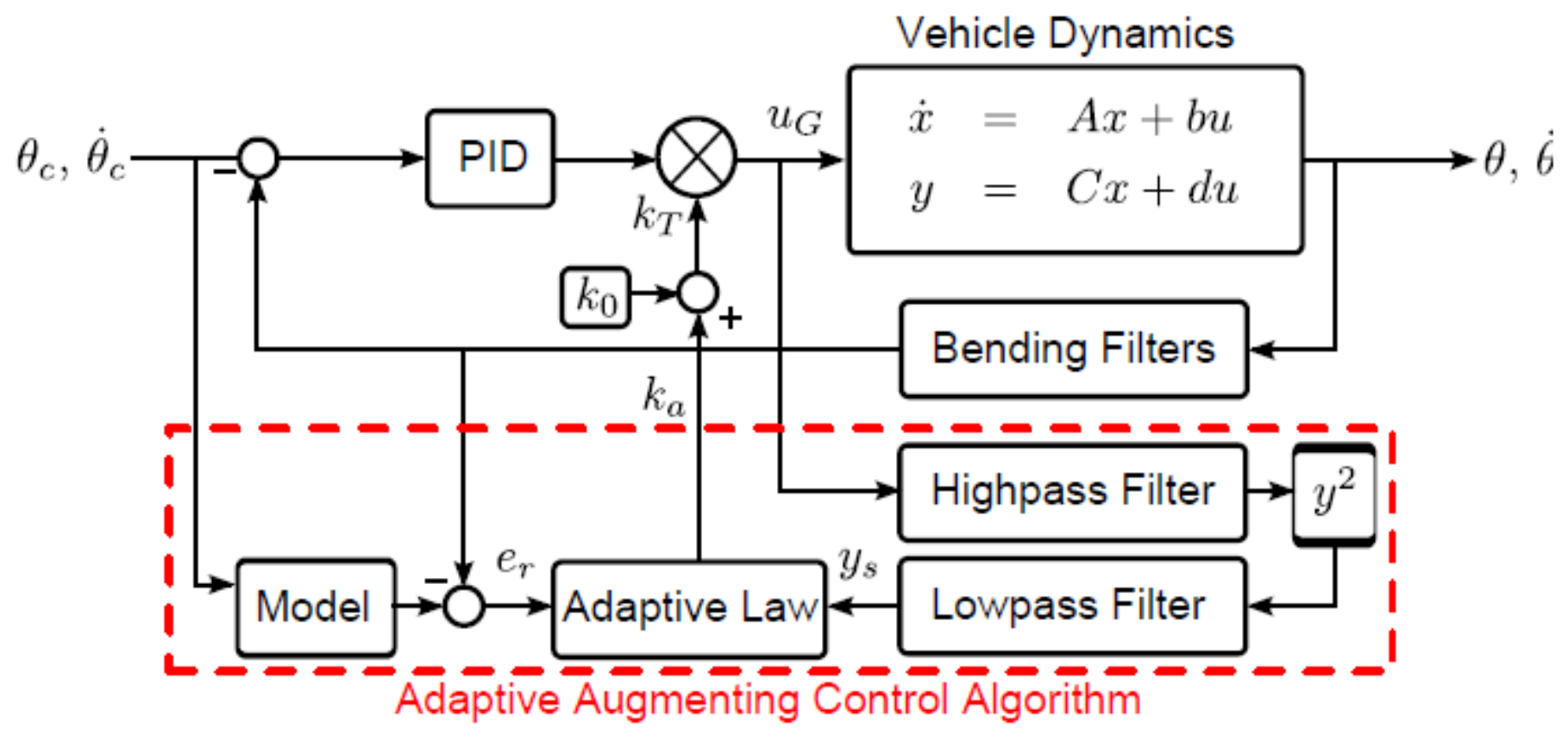




\section{Adaptive Update Law}

- Design features of augmenting signal update law

- Respond to error in ability of vehicle to track commands

- Respond to undesirable control-structure interaction

- Return to baseline control design when not needed

- Consider the update law

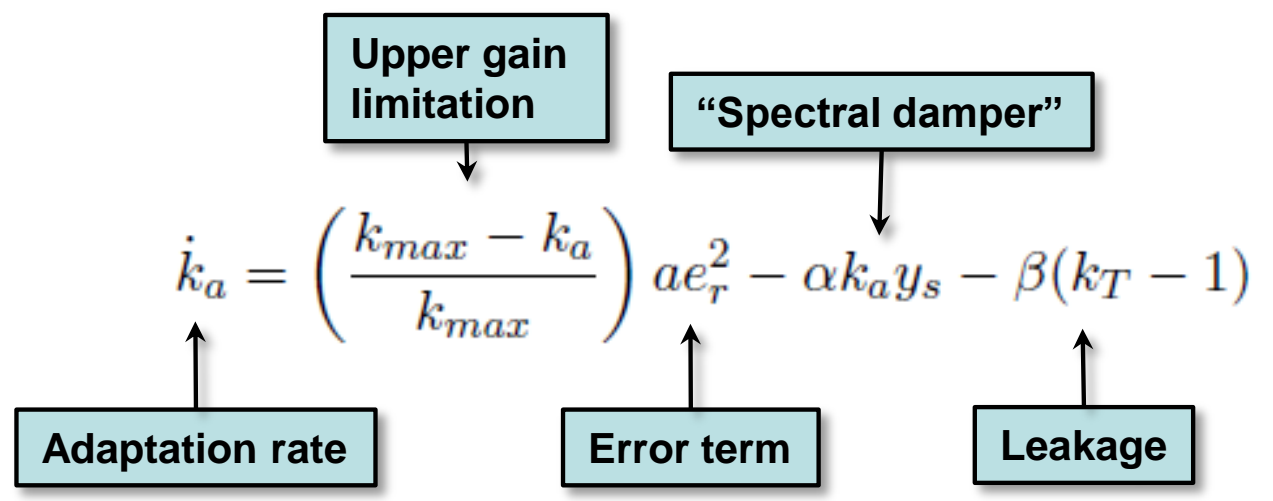

$$
k_{T}=k_{0}+k_{a}
$$




\section{Update Law: Error Term}

\section{- Protect baseline control gains (avoid undesirable adaptation)}

- Adaptive Augmenting Control

- Model reference architecture uses the difference between the vehicle states and a reference model to drive the adaptive update law

- Reference model is designed to simulate the desired closed loop performance. For simplicity, this was selected to be a second order system that tracks the guidance commands:

$$
\left[\begin{array}{c}
\dot{\theta}_{r} \\
\ddot{\theta}_{r}
\end{array}\right]=\left[\begin{array}{cc}
0 & 1 \\
-\omega_{r}^{2} & -2 \zeta_{r} \omega_{r}
\end{array}\right]\left[\begin{array}{c}
\theta_{r} \\
\dot{\theta}_{r}
\end{array}\right]+\left[\begin{array}{cc}
0 & 0 \\
\omega_{r}^{2} & 2 \zeta_{r} \omega_{r}
\end{array}\right]\left[\begin{array}{c}
\theta_{c} \\
\dot{\theta}_{c}
\end{array}\right]
$$

- The reference model is explicitly discretized with a scheduled natural frequency and damping

$$
\gamma_{r}=\theta_{r}-\theta
$$

Reference Model State

\section{- Increase robustness; prevent or delay LOV}

- Standard adaptive update law with $e_{r}=c \gamma_{r}+\dot{\gamma}_{r} \quad$ (mixed attitude/rate error)

$$
\dot{k}_{a}=\left(\frac{k_{\max }-k_{a}}{k_{\max }}\right) a e_{r}^{2}-\alpha k_{a} y_{s}-\beta\left(k_{T}-1\right)
$$




\section{Update Law: Leakage}

- Protect baseline control gains (avoid undesirable adaptation)

- Leakage

- Provides automatic re-convergence to the total loop gain of the nominal control system

$$
\dot{k}_{a}=\left(\frac{k_{\max }-k_{a}}{k_{\max }}\right) a e_{r}^{2}-\alpha k_{a} y_{s}-\beta\left(k_{T}-1\right)
$$

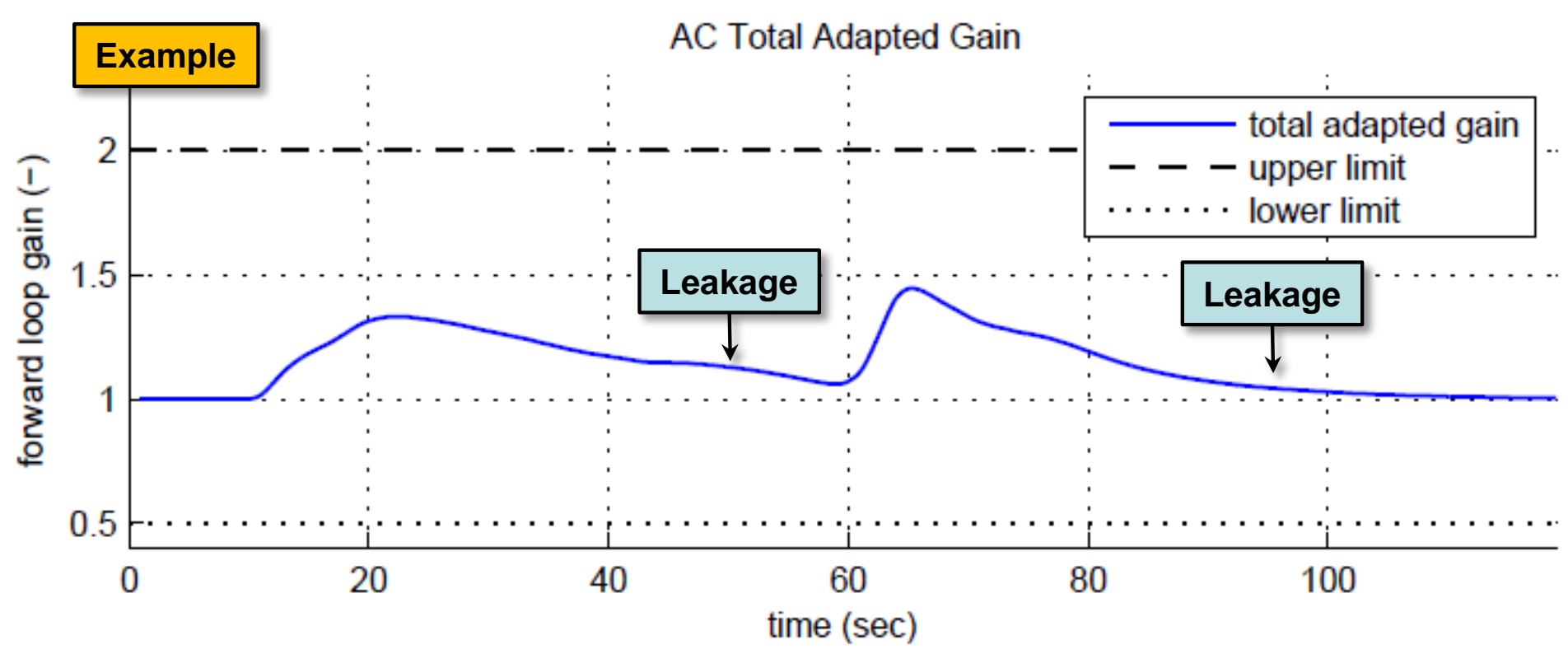




\section{Update Law: Spectral Damper}

- Spectral damper term

$$
\begin{aligned}
y_{H P} & =H_{H P}(s) u_{G} \\
y_{s} & =H_{L P}(s) y_{H P}^{2}
\end{aligned}
$$

- Spectral damper signal $y_{s}$ is a rectified band-pass/high-pass signal capturing undesirable high-frequency dynamics in the control loop

- Gain decay is proportional to magnitude of this signal

- Provides sensing and mitigation of control-structure interaction

$$
\dot{k}_{a}=\left(\frac{k_{\max }-k_{a}}{k_{\max }}\right) a e_{r}^{2}-\alpha k_{a} y_{s}-\beta\left(k_{T}-1\right)
$$
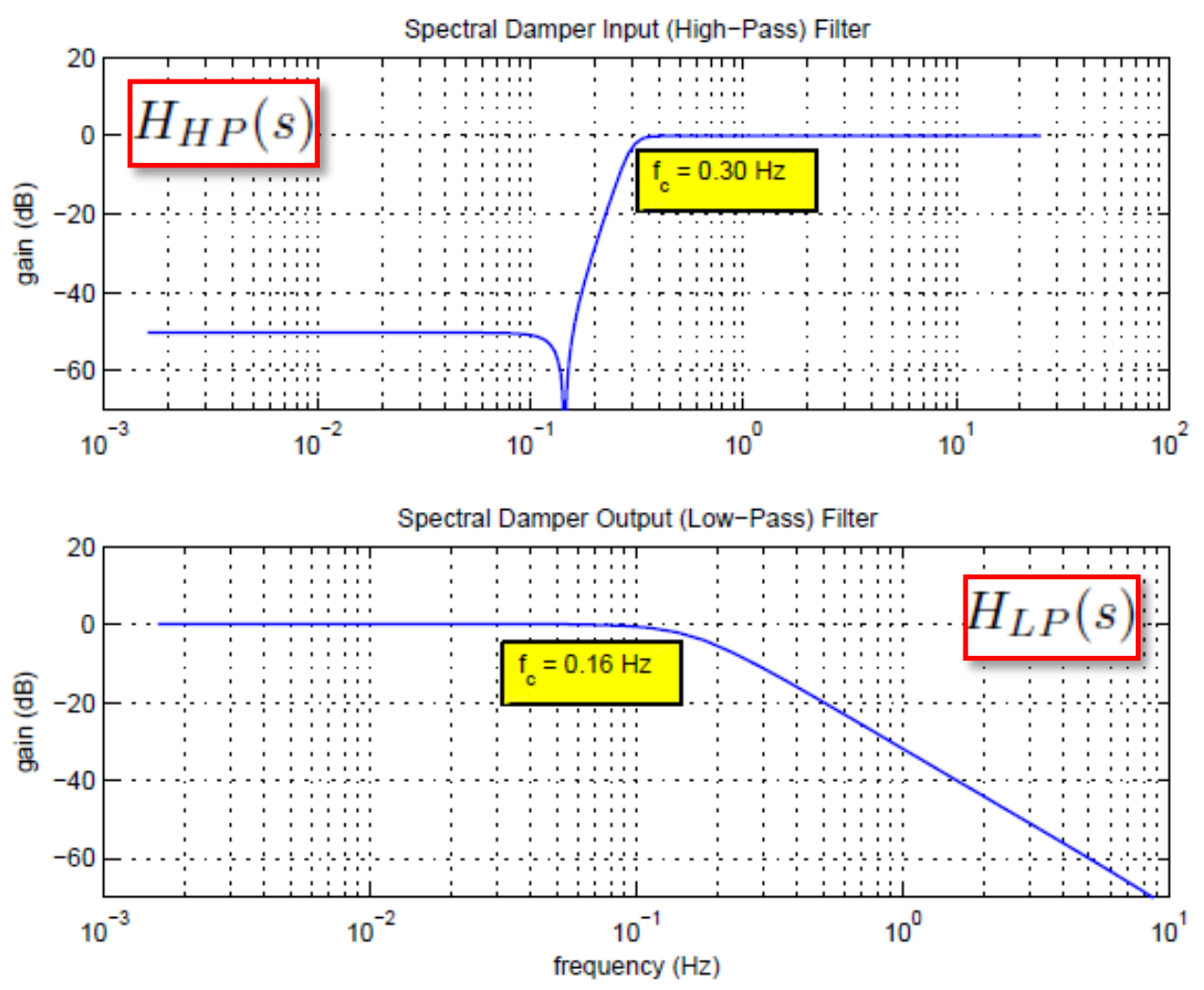


\section{Design of Spectral Damper Filters}

- High-frequency closed-loop spectrum under high forward loop gain can be readily deduced from the open-loop frequency response

- Correlation allows design of spectral damper filters

- Used directly to determine high-pass cutoff frequency specification
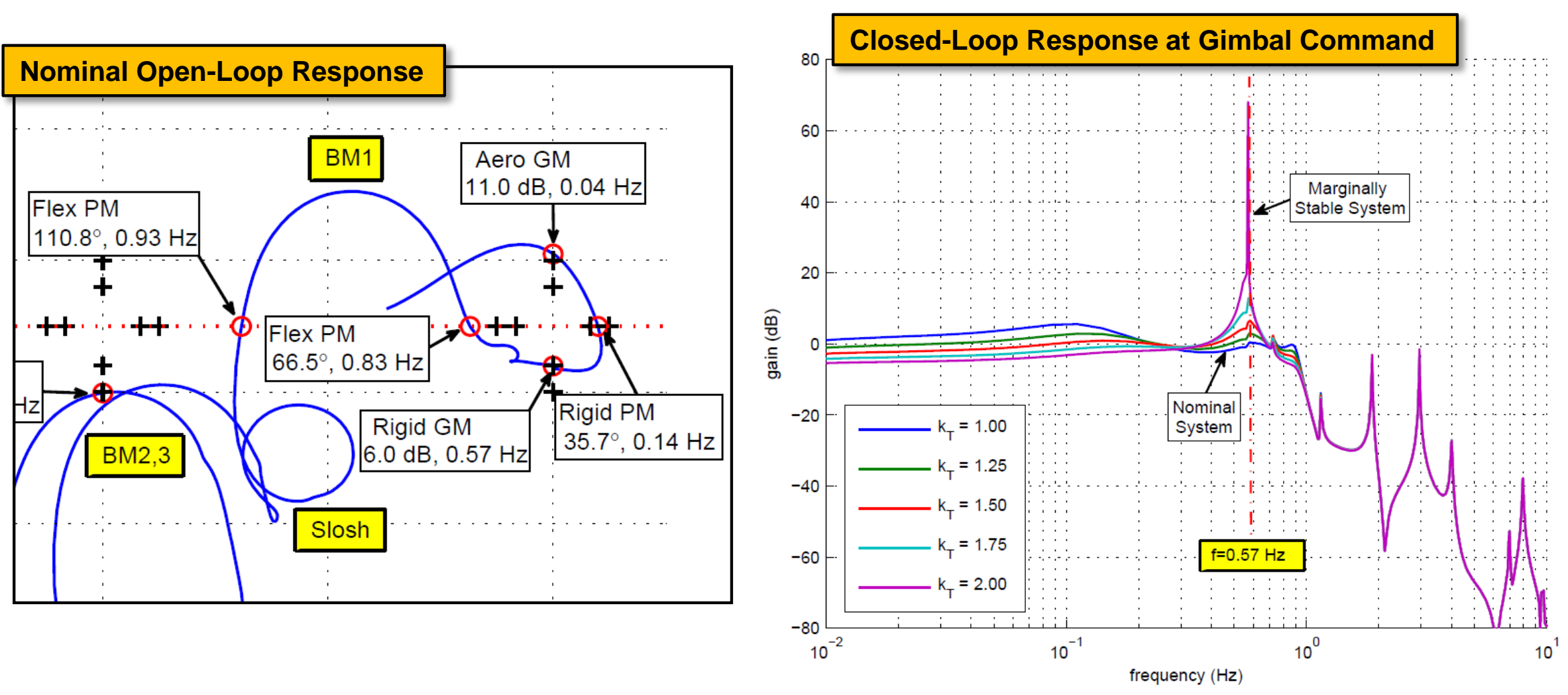
- Simulation based on representative launch vehicle data

- High-fidelity linearized pitch dynamics along first stage open-loop trajectory

- Time-varying model with reduced FCS (PD+rate filter)

- 20+ flex modes + 4 slosh modes, winds, DTV, aeroelastic models

- Gimbal angle and rate limits

- Matrix test cases selected to explore the design philosophy

1. Minimal adaptation for nominal vehicle

2. Improved performance for dispersions within baseline controller capability

3. Prevent/delay LOV for dispersions outside baseline controller capability

- Examples: Heavy/slow, light/fast, low slosh damping, gain-loading error, aerodynamic instability, bending model error, etc.

- Single gain set for all test cases and flight conditions

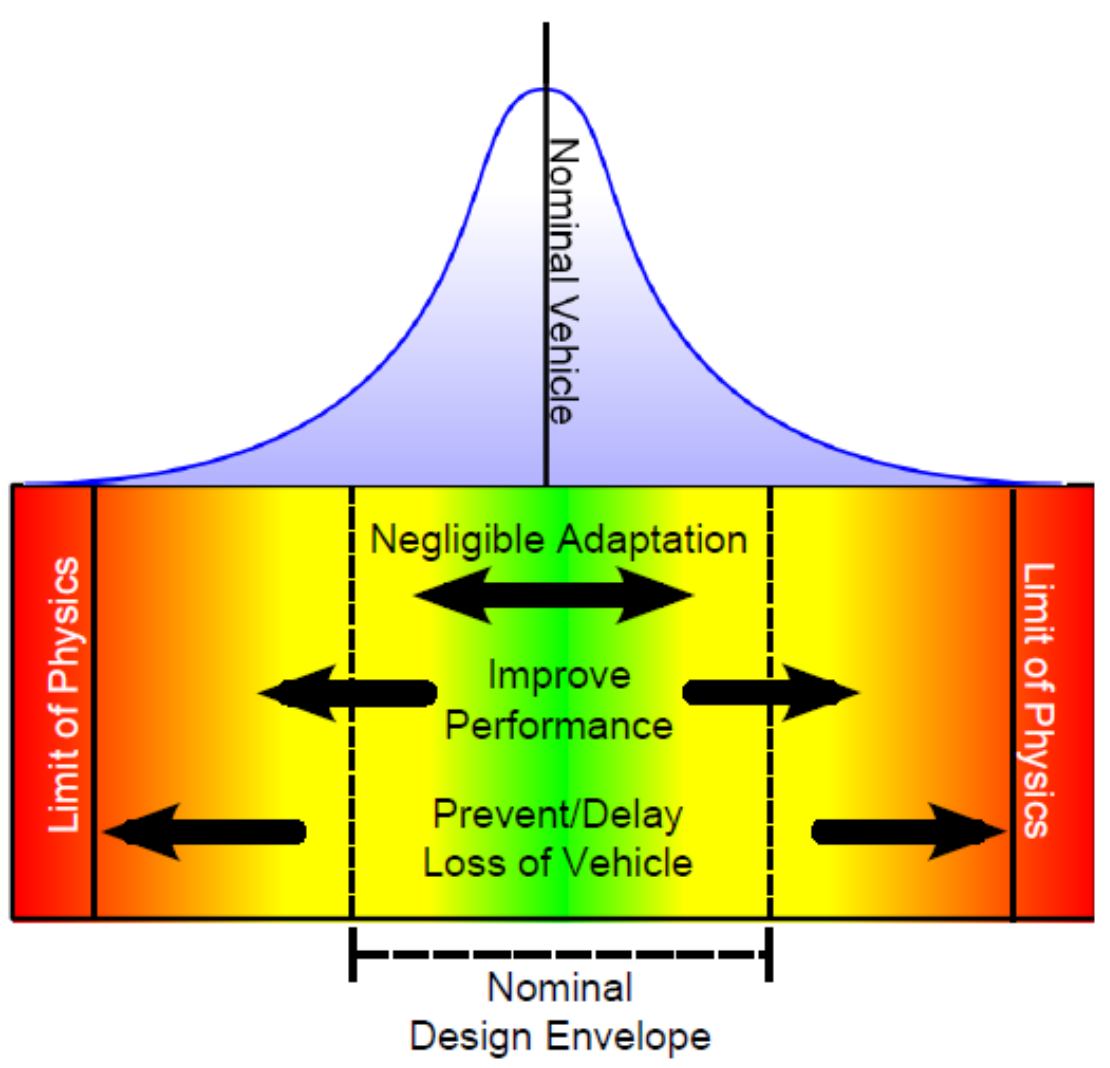




\section{Test Case 1: Nominal System}

\section{- For nominal plant and environment, we expect minimal adaptation}

- Leakage effect in absence of external factors should attract gain to unity

- Negligible adaptation is indicative of good reference model parameter selection

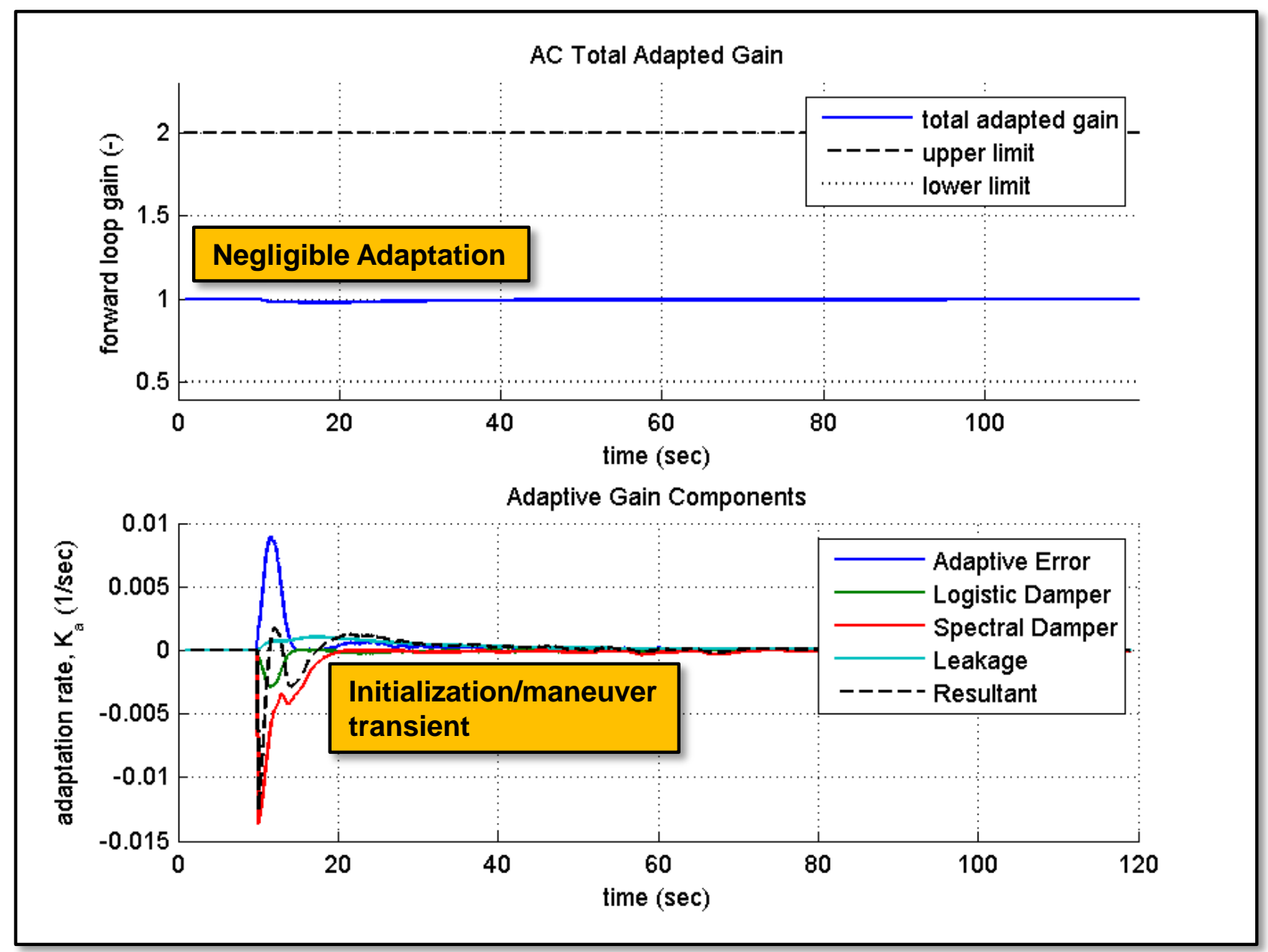




\section{Test Case 1: Nominal System}

- At total forward loop gain of 1, control system converges to baseline

- Rigid body performance metrics are nearly identical for the nominal vehicle

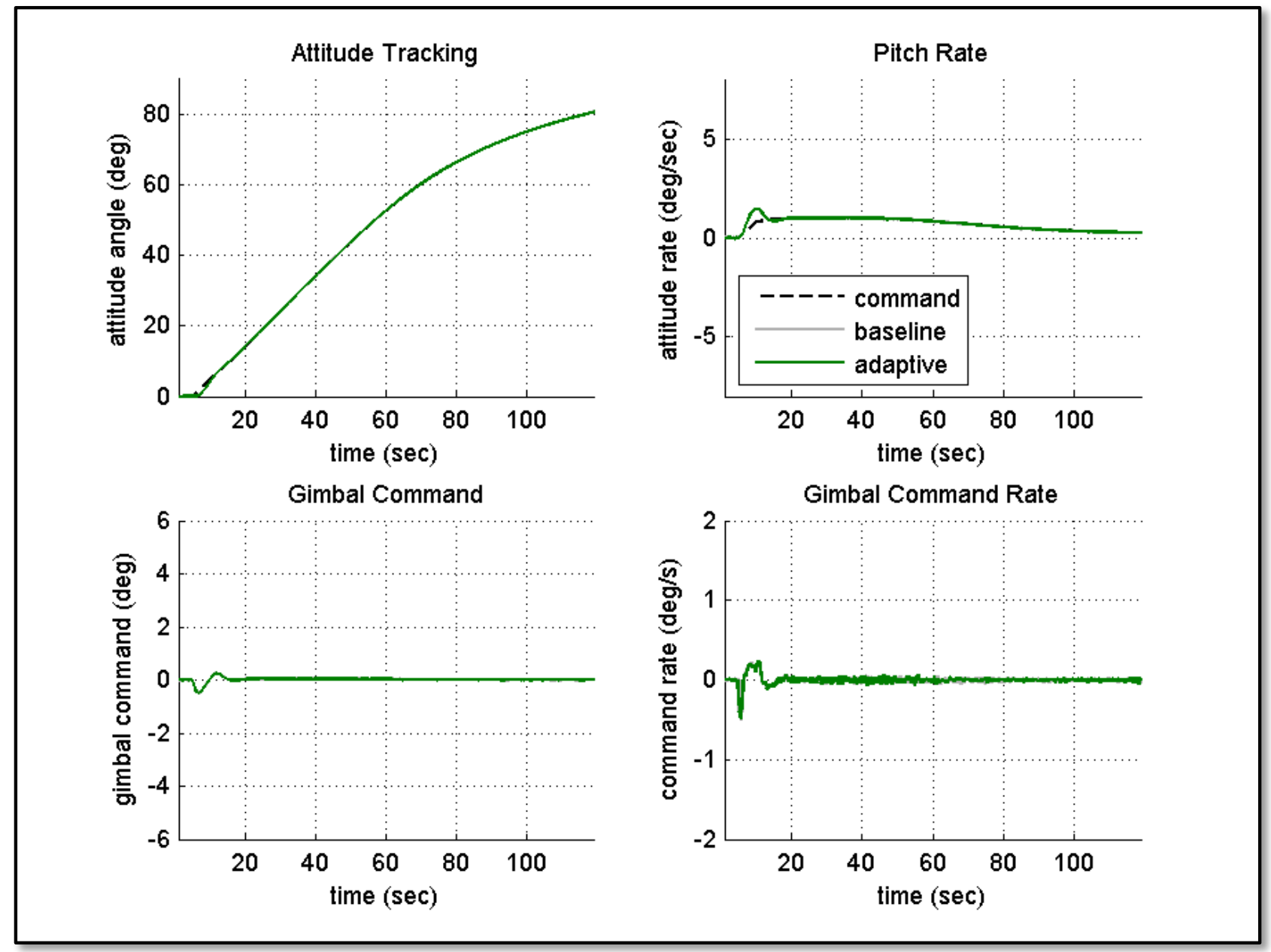




\section{Test Case 1: Nominal System}

- Frequency-domain stability margins are not impacted by adaptation; total loop gain remains the same

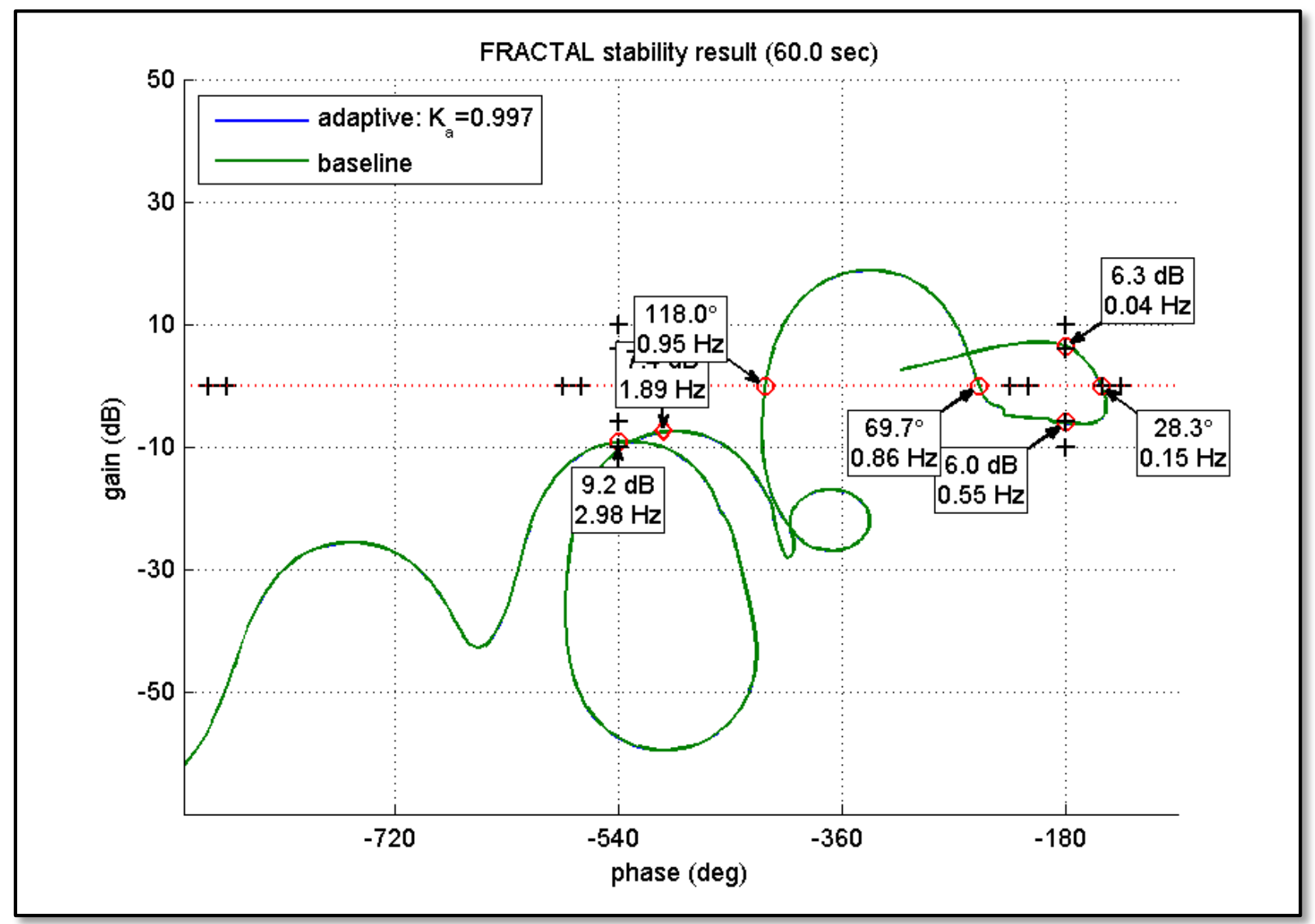




\section{Test Case 9: Aerodynamic Instability}

- Simulated aerodynamic modeling error $+3 \sigma$ wind shear

- Winds profile derived from bad single case wind shear at max $Q$

- 3x increase in Cza, aerodynamic moment coefficient slope

- The nominal vehicle is already unstable at $\max Q$

- Wind shear initiates static aerodynamic divergence; adaptive control recovers

- Baseline controller induces excessive qa; does not recover before loss of vehicle

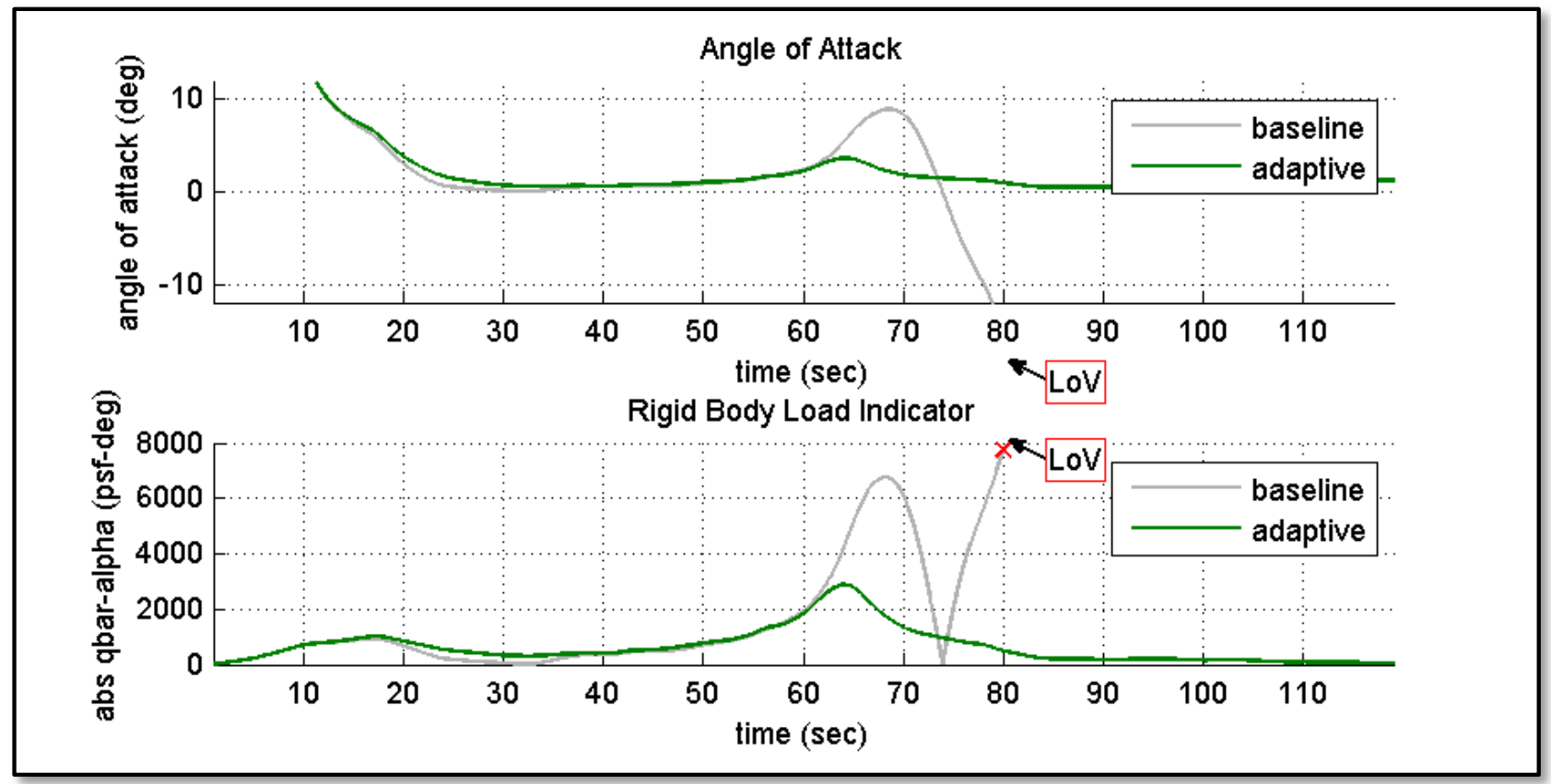




\section{Test Case 9: Aerodynamic Instability}

- Nominal controller encounters gimbal saturation and diverges

- Vehicle without adaptive controller exceeds load limit of 8500 psf-deg

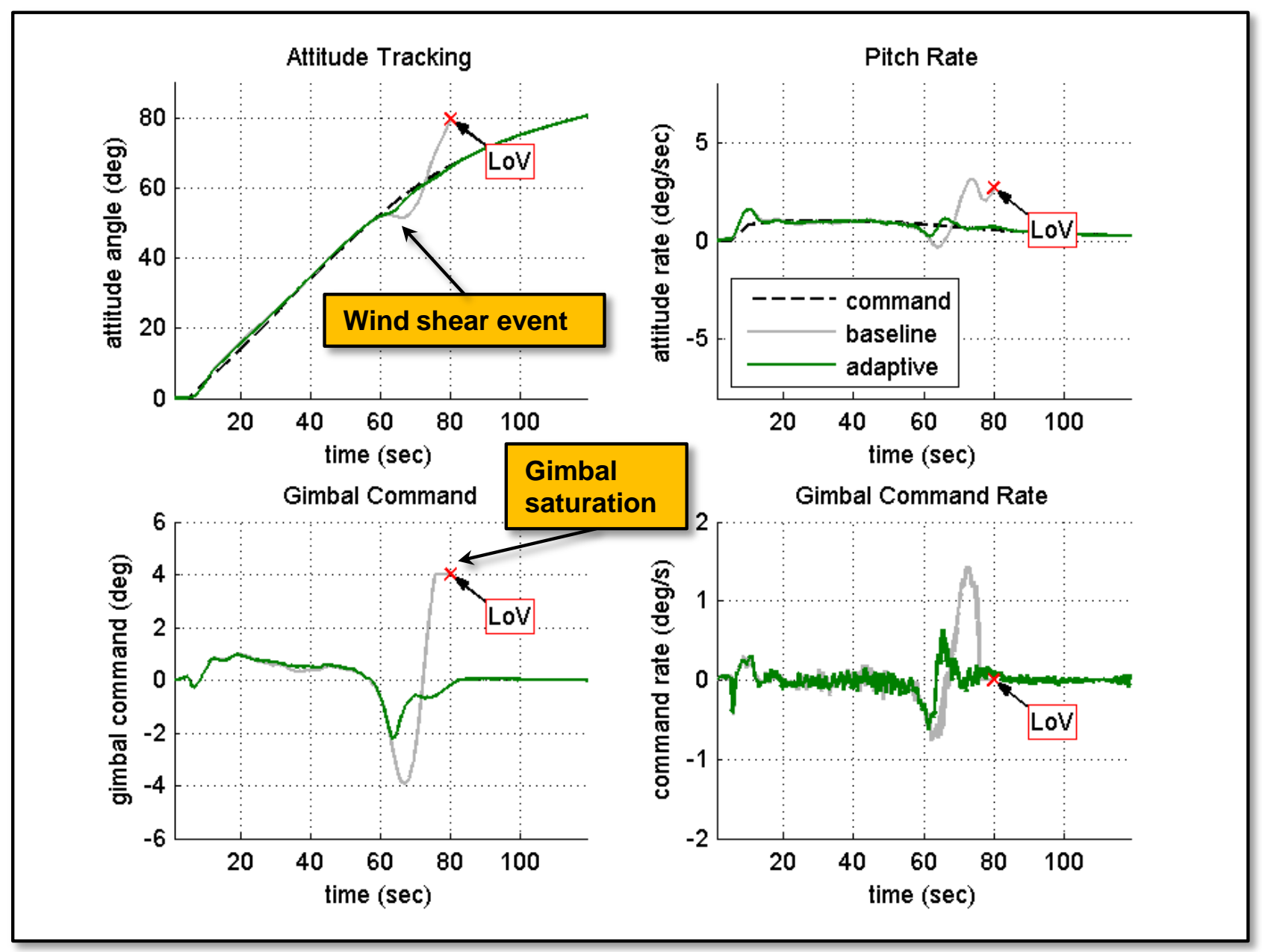




\section{Test Case 9: Aerodynamic Instability}

- Adaptation maintains vehicle at marginal stability limit until excited by shear

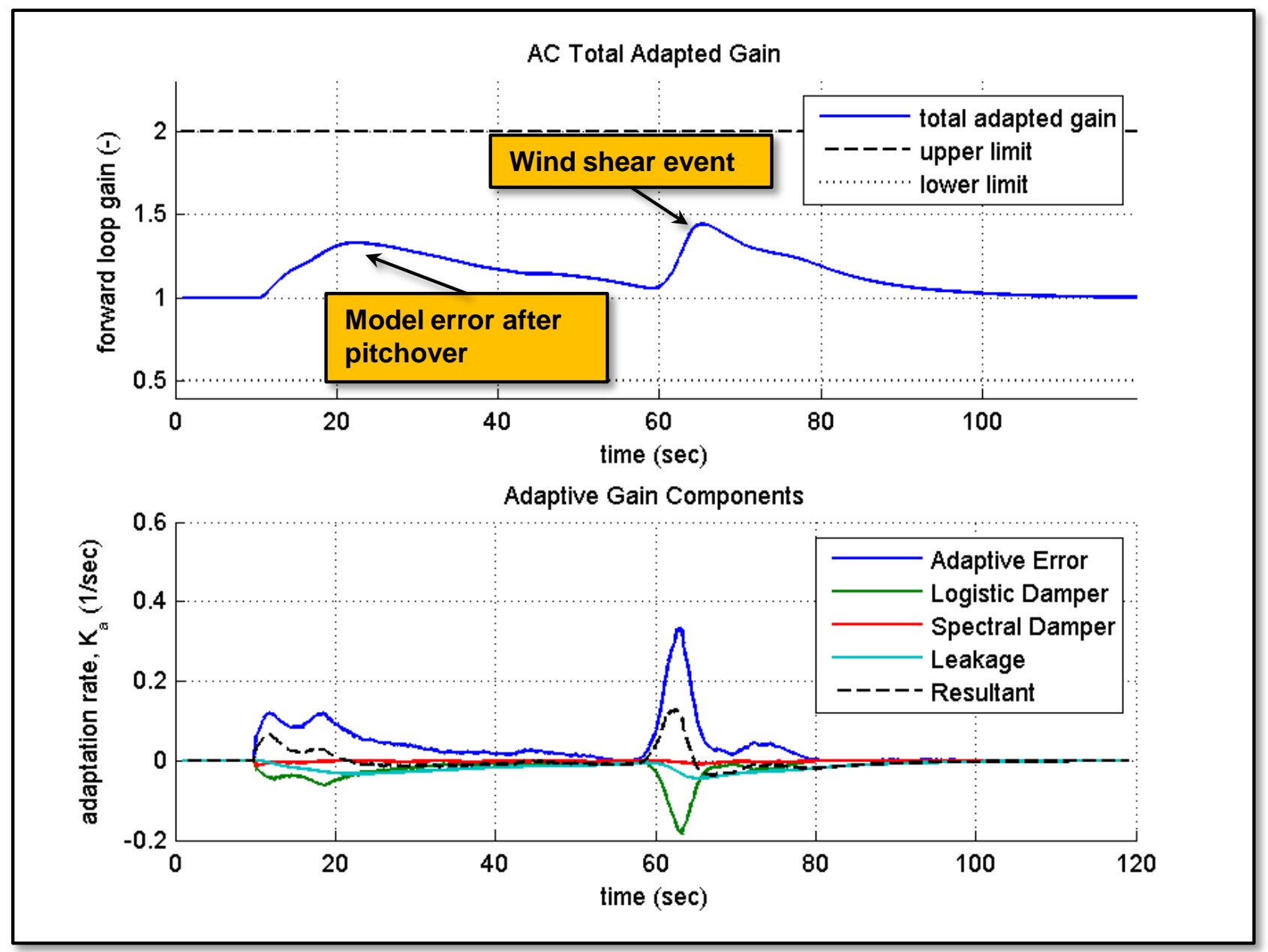




\section{Test Case 9: Aerodynamic Instability}

- Examination of loop gain reveals effect on open-loop stability characteristics

- Dynamic and responsive increase in gain is able to retain stability in an LTI sense

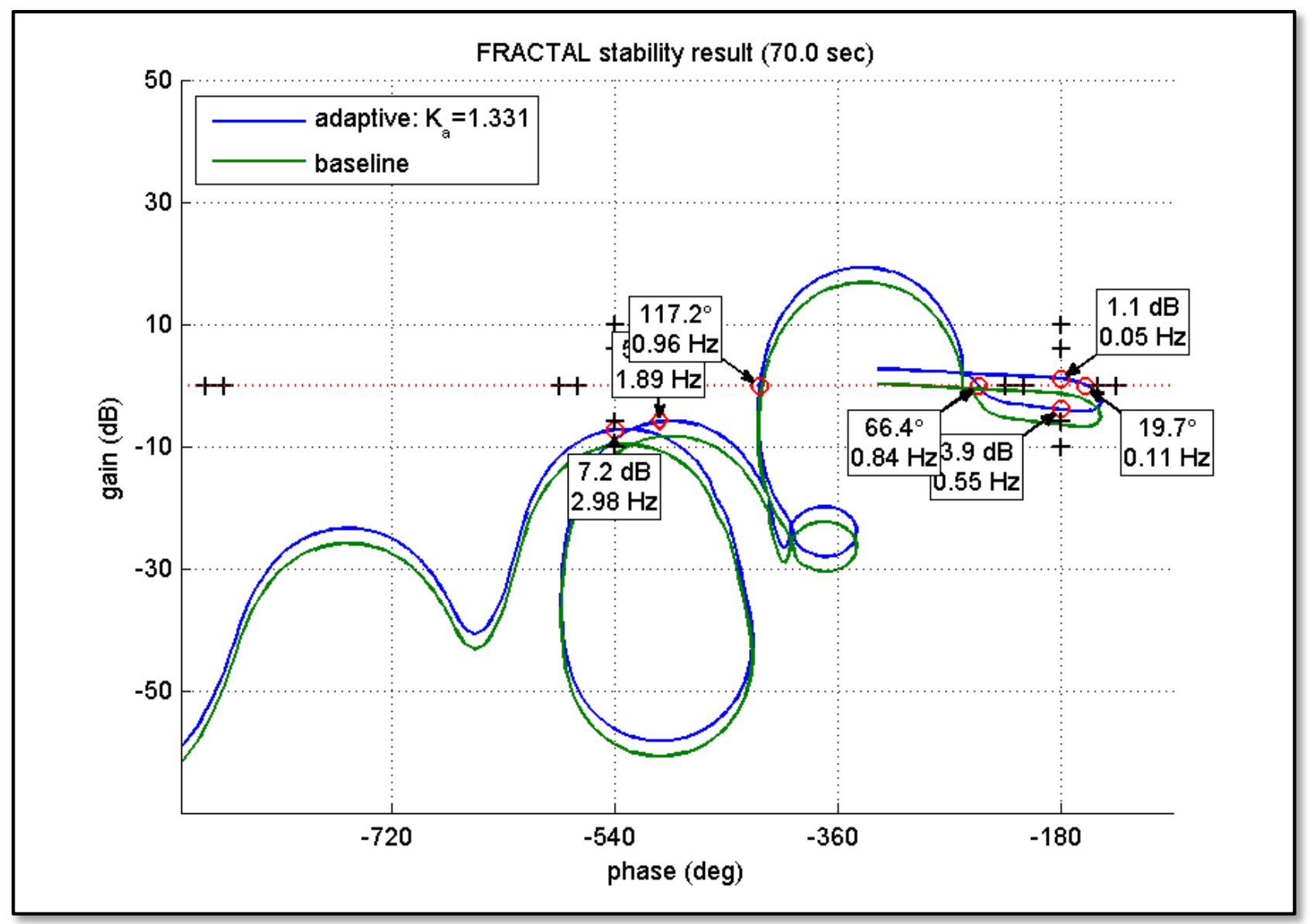




\section{Test Case 10: Unstable Bending}

- Nominal vehicle is simulated with $-30 \%$ frequency error $+6 \mathrm{~dB}$ modal gain increase in first lateral bending mode

- Frequency response of open-loop plant predicts BM1 instability

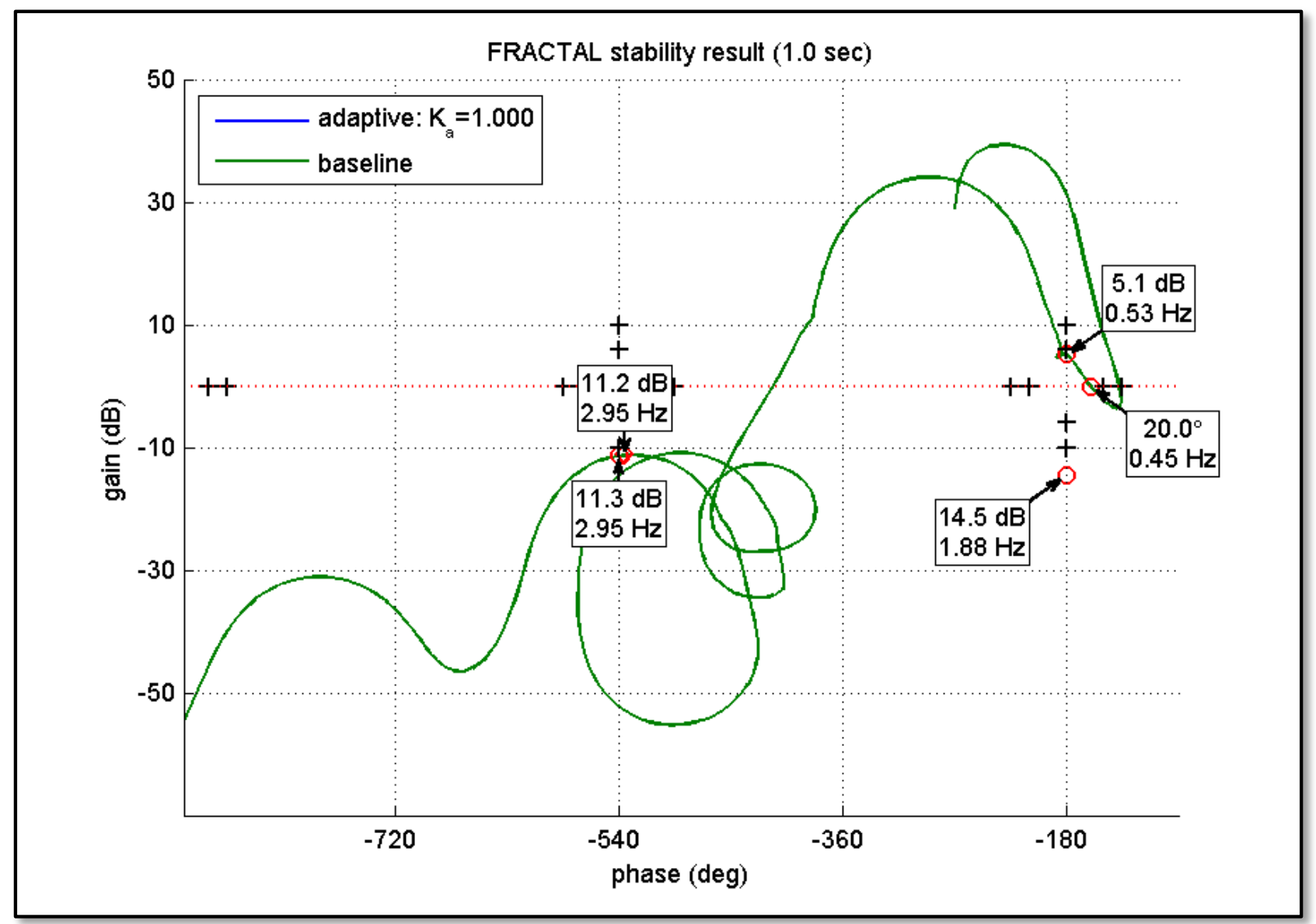




\section{Test Case 10: Unstable Bending}

- Spectral damping filter detects and responds to bending resonance

- Dominant factor due to spectral damping effect drives gain down to recover stability of $1^{\text {st }}$ bending mode; error and leakage drive recovery

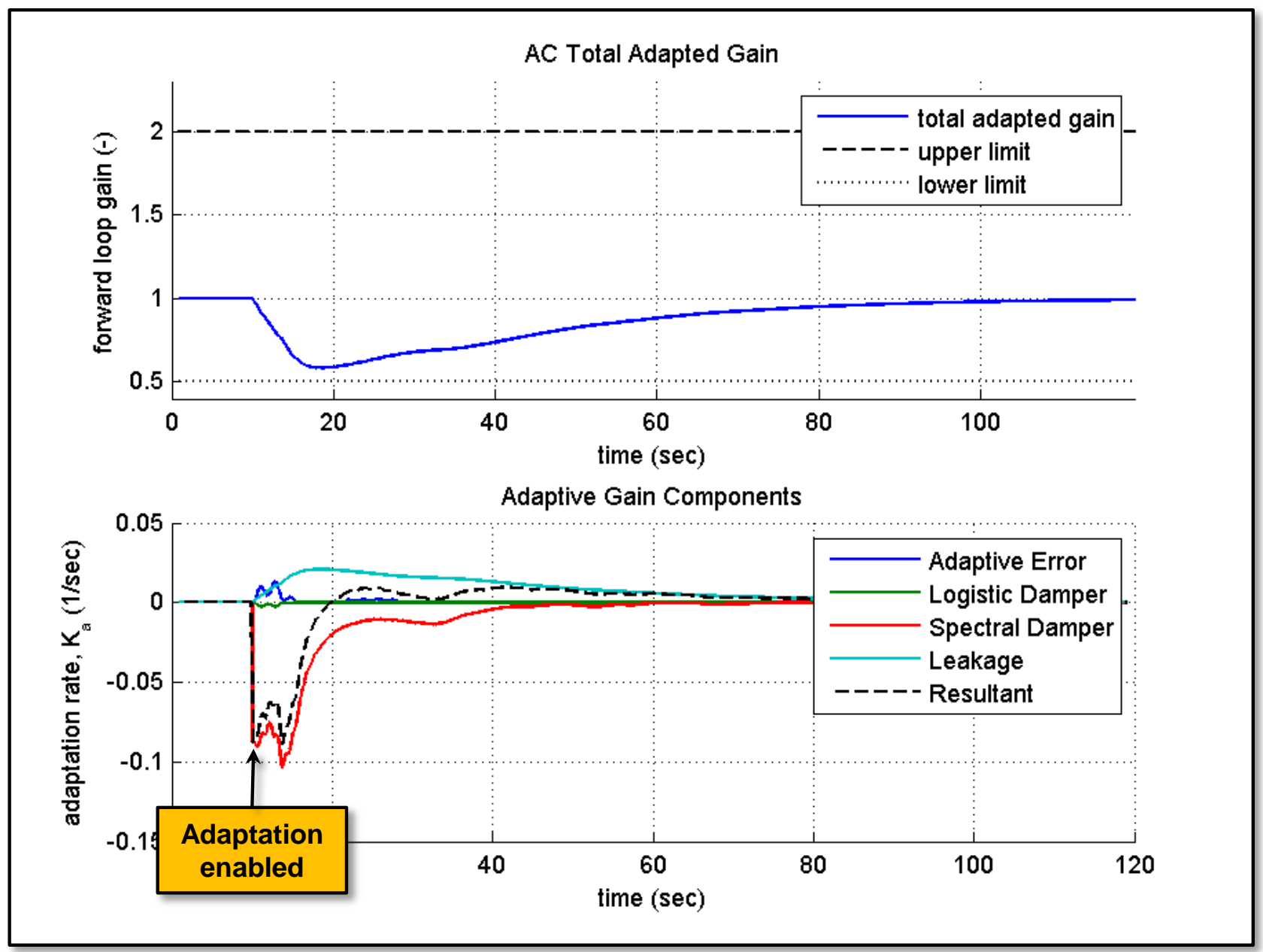




\section{Test Case 10: Unstable Bending}

- Adaptive controller reduces gain to bring BM1 to stable limit cycle

- BM1 remains marginally stable throughout flight and system slowly recovers lost performance as BM1 shifts up in frequency due to propellant consumption

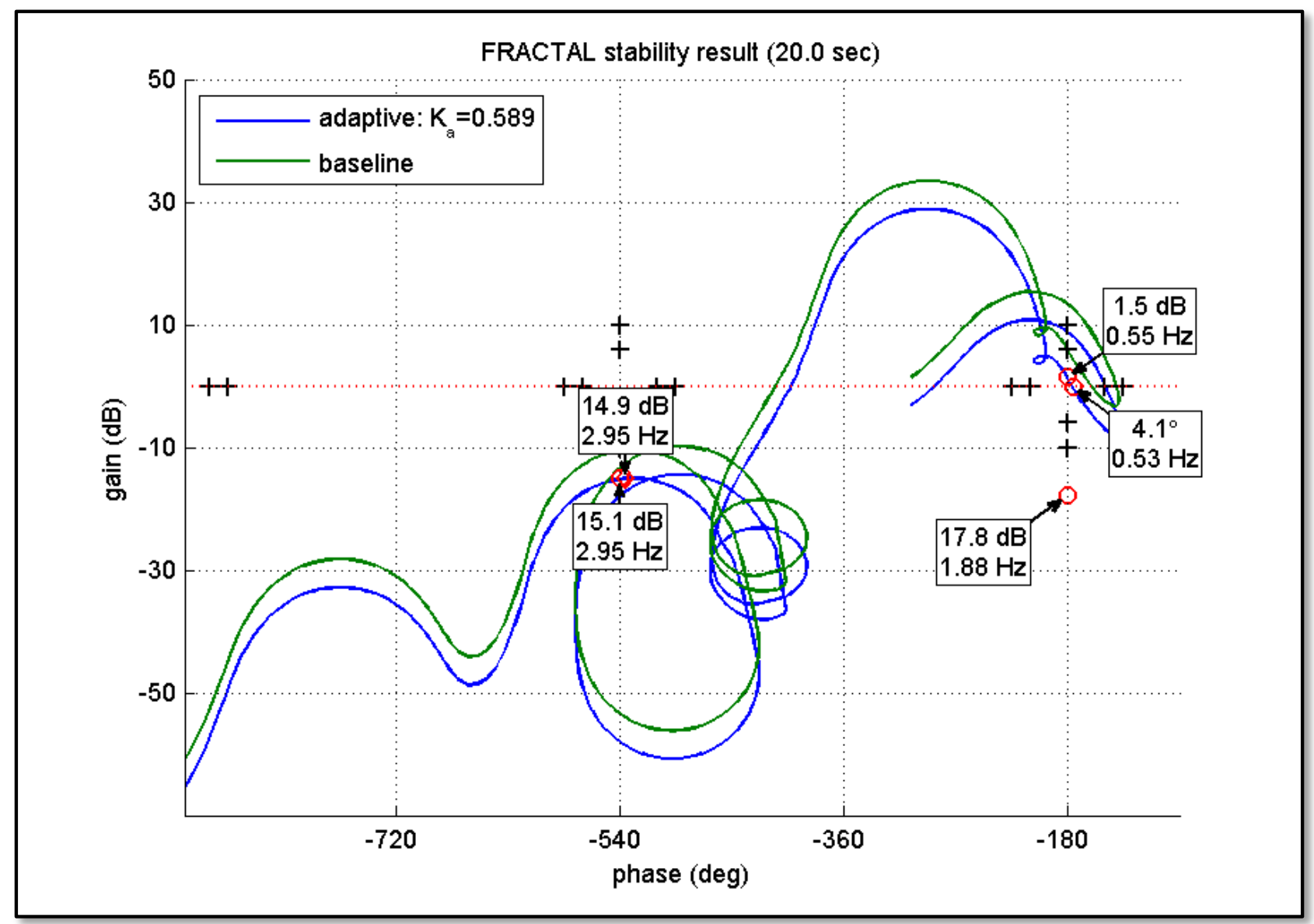




\section{Test Case 10: Unstable Bending}

- Baseline controller induces structural failure due to resonance

- Arbitrary limit of strain energy at $1 \mathrm{E} 4 \mathrm{ft}-\mathrm{lbf}$ (dependent on retained modal mass)

- Continued simulation results in divergence of first bending mode

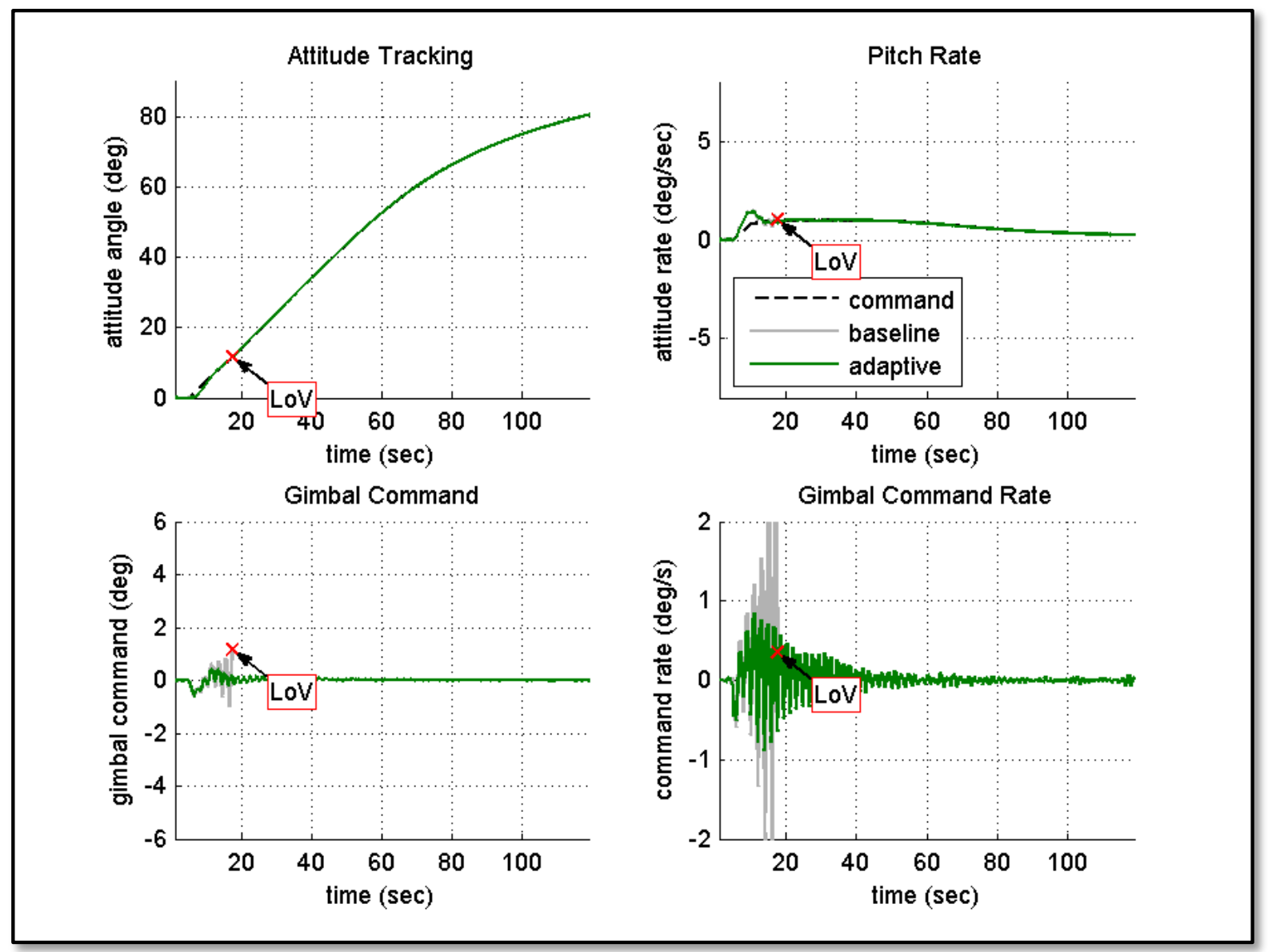




\section{Test Case 10: Unstable Bending}

\section{- Spectral damper detail}

- High-pass filter captures BM1 frequency in gimbal command

- Spectral damper signal attenuates gain

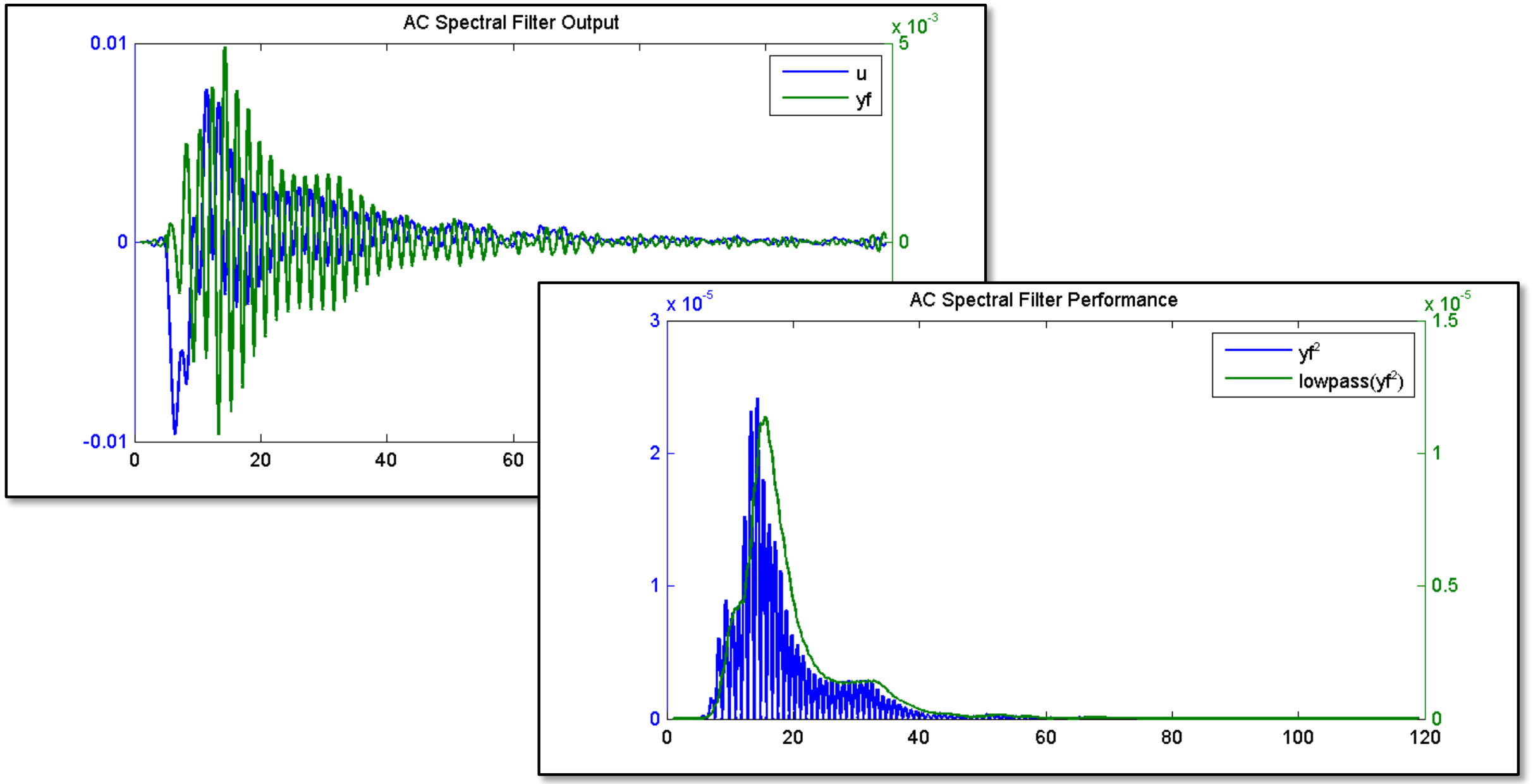




\section{Summary and Forward Work}

\section{- Summary}

- The present design provides a straightforward, easily understood approach to online forward loop gain adaptation that is cognizant of the real features of conditionally stable flexible launch vehicle dynamics

- The system is attracted to the nominal design and does not require persistent excitation

- Augmentation has the capability to reduce loop gain below unity to recover in the presence of adverse control structure interaction

- Method of design of filters and limits follows directly from classical concepts

\section{- Forward work}

- The present method of stability assessment, based on frequency-domain analysis, is reasonable but not mathematically rigorous

- It may be possible to fit the present architecture into the circle criterion for an LTI plant

- Adopting the circle criterion as a design metric restricts the nominal performance

- Restrictions may preclude its application (plant is LTV, not SPR, and non-minimum phase)

- Adaptation gain design is more or less trial-and-error $\rightarrow$ a formal methodology is required 